Algebraic $\& \mathcal{G}$ Geometric Topology

Volume 2 (2002) 757-789

Published: 2 October 2002

ATG

\title{
Floer homology of surgeries on two-bridge knots
}

\author{
JACOB RASMUSSEN
}

Abstract We compute the Ozsváth-Szabó Floer homologies $H F^{ \pm}$and $\widehat{H F}$ for three-manifolds obtained by integer surgery on a two-bridge knot.

AMS Classification 57R58; 57M27

Keywords Floer homology, two-bridge knot

\section{Introduction}

The groups $H F^{ \pm}$introduced by Ozsváth and Szabó in [6], [7] have shed new light on our understanding of Floer homology for three-manifolds. Conjectured to be equal to the equivariant Seiberg-Witten Floer groups, the Ozsváth-Szabó groups have most of the known properties of these groups, as well as some (such as the exact triangle) which were only conjectured. In addition, they appear to be more computable than the Seiberg-Witten Floer groups; at the least, there is an algorithmic procedure to find the generators for the chain complex associated to a given three-manifold. On the other hand, there are some potential stumbling blocks: the size of the chain complex is typically much larger than that of the associated homology groups, and the differentials in the complex can be difficult to determine.

In this paper, we compute the Ozsváth-Szabó Floer homology for integral surgeries on two-bridge knots. Although these are some of the simplest available three-manifolds, the fact that the corresponding Seiberg-Witten Floer groups are still unknown indicates the computational effectiveness of the OzsváthSzabó groups. Moreover, the method of calculation provides some grounds for optimism: the formal properties of their chain complex enable us to compute its homology without having to understand all of its differentials.

The result of our computation may be summarized as follows: all the Floer homologies associated to a two-bridge knot $K$ are determined by two classical invariants of the knot - the Alexander polynomial and the signature $\sigma(K)$. For the moment, we restrict ourselves to describing some interesting special cases. 
Theorem 1 Let $K$ be a two-bridge knot and $T$ the $(2,2 n+1)$ torus knot of the same signature. Let $K^{0}$ and $T^{0}$ be their 0 -surgeries. Denote by $\mathbf{s}_{k}$ the $\operatorname{Spin}^{c}$ structure on $K^{0}$ with $c_{1}\left(\mathbf{s}_{k}\right)=2 k$. Then $H F^{+}\left(K^{0}, \mathbf{s}_{k}\right) \cong Q \oplus H F^{+}\left(T^{0}, \mathbf{s}_{k}\right)$, where $Q$ is a free $\mathbf{Z}$ module concentrated in a single grading.

As a corollary, we find that the Ozsváth-Szabó analogue of Frøyshov's $h$-invariant is determined by the signature:

Corollary 1 Let $K$ be a two-bridge knot and $K^{1}$ the manifold obtained by 1-surgery on it. Then $d\left(K^{1}\right)=\min (0,-2\lceil\sigma(K) / 4\rceil)$.

We now give a quick overview of the calculation. The first two sections of the paper are an elaboration of section 8 of [6], which computes $H F^{ \pm}$and $\widehat{H F}$ for the simplest two-bridge knots - the $(2, n)$ torus knots. First, any surgery on a two-bridge knot $K$ admits a natural Heegaard splitting of genus 2. This is described in section 2. In section 3, we consider $n$ surgery on $K$ for $n \gg 0$. In this case, the generators of the chain complex $\widehat{C F}\left(K^{n}\right)$ are particularly easy to write down. Using certain "annular differentials" described in [6], we compute the grading in this complex. In section 4, we use these annular differentials to compute $\widehat{H F}\left(K^{n}\right)$ for a particular Spin $^{c}$ structure. We next consider the complex $\mathrm{CF}^{+}$. Although we do not know what most of the differentials in this complex are, its symmetries allow us to compute $\mathrm{HF}^{+}$in every $\operatorname{Spin}^{c}$ structure, using only the one group we computed directly. In section 5 we apply the exact triangle of [7] to get $\mathrm{HF}^{+}\left(K^{n}\right)$ for any $n \in \mathbf{Z}$. Finally, in section 6 we briefly discuss the extent to which the methods of this paper apply to other knots.

The author would like to thank Peter Kronheimer for his advice and support, Kim Frøyshov for helpful discussions regarding the $h$-invariant, and Peter Ozsváth and Zoltán Szabó for their interest in the subject.

\section{Two-bridge knots}

We begin by reviewing some classical facts about two-bridge knots. Good references for this material are [5] and [1]. First, the definition: a two-bridge knot is any knot $K$ which admits a presentation of the form shown in Figure 1: two maxima, two minima, and a braid $B_{K}$ in between. If we split $S^{3}$ along a horizontal plane, we can write the pair $\left(S^{3}, K\right)$ as the union of two copies of $\left(B^{3}, \ell_{1} \cup \ell_{2}\right)$, where $\ell_{1}$ and $\ell_{2}$ are a pair of parallel line segments. The two pieces are glued together by the element of the mapping class group of $S^{2}$ with four marked points which corresponds to the braid $B_{K}$. 


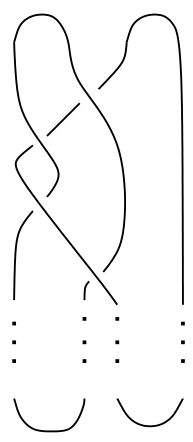

Figure 1: A two-bridge knot

Since the double cover of $B^{3}$ branched along $\ell_{1} \cup \ell_{2}$ is a solid torus, the double cover of $S^{3}$ branched along $K$ is a lens space. The basic fact about two bridge knots is that they are classified by their branched double covers.

Proposition 2.1 (Schubert) For every oriented lens space $L(p, q)$ with $p$ odd, there is a unique two-bridge knot $K(p, q)$ with branched double cover $L(p, q)$.

\section{Remarks}

(1) The spaces $L(p, q)$ with $p$ even are obtained as branched double covers of two-bridge links.

(2) Lens spaces which are orientation preserving diffeomorphic have the same knot, so that e.g. $K(p, q)=K\left(p, q^{\prime}\right)$ if $q^{\prime} \equiv q^{-1} \bmod p$. Lens spaces which are orientation reversing diffeomorphic correspond to mirror image knots. In particular, $K(p, q)$ is amphichiral if and only if $q^{2} \equiv-1 \bmod p$.

(3) The braid $B_{K(p, q)}$ can be obtained from a continued fraction expansion of $p / q$, as explained in [5].

Some simple examples of two-bridge knots include the left-hand $(2, p)$ torus knots, which are $K(p, 1)$ in our notation, and the twist knots $K(p, 2)=K(p$, $(p+1) / 2)$.

\section{$2.1 \quad$ Schubert normal form}

The knot $K(p, q)$ admits a canonical projection known as the Schubert normal form, which is particularly well-adapted to our purposes. In this section, we 


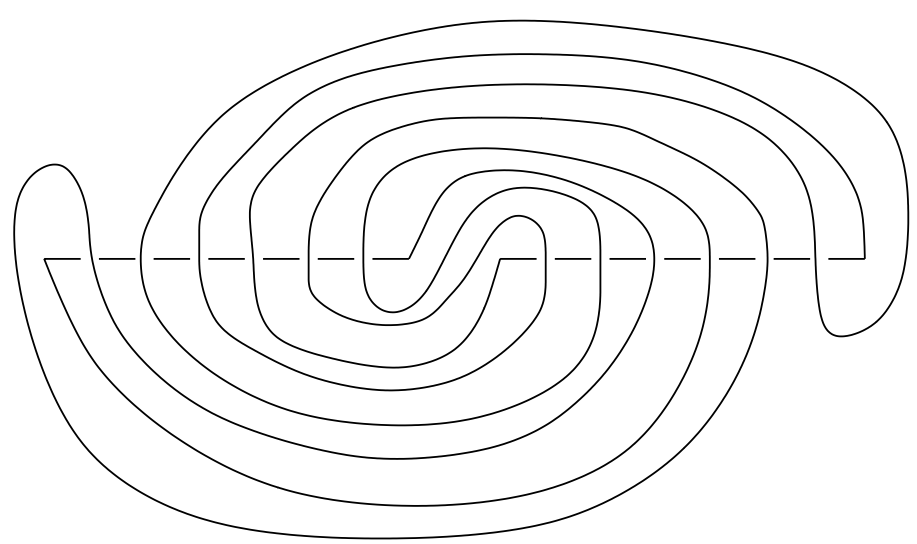

Figure 2: The Schubert normal form of $K(7,3)$

describe this form and its relevant properties. Proofs may be found in [1] or [9].

Consider the knot $K(p, q)$. Without loss of generality, we can assume $-p<$ $q<p$ and that $q$ is odd. The normal form of $K(p, q)$ is a projection of the sort shown in Figure 2. We break $K(p, q)$ into 4 segments: two underbridges $U_{1}$ and $U_{2}$ (drawn horizontally) and two overbridges $O_{1}$ and $O_{2}$. If we travel along one of the $U_{i}$, we go through $p-1$ undercrossings, which alternate between $O_{1}$ and $O_{2}$. Similarly, if we travel along one of the $O_{i}$, we go through $p-1$ overcrossings, alternating between $U_{1}$ and $U_{2}$.

To give a precise description, we consider small neighborhoods of the underbridges. One such neighborhood is shown in Figure 3. Abstractly, it is a disk with marked points $a_{0}, a_{1}, \ldots a_{2 p-1}$ on its boundary. To get the Schubert normal form, we glue these two disks together by an orientation reversing diffeomorphism of $S^{1}$ which identifies the point $a_{i}$ on one disk with the point $a_{q-i}$ on the other. (All the labeling is modulo $2 p$.) The resulting diagram is most naturally thought of as living on a sphere, but if we want to draw it, we project onto a plane.

The Schubert normal form is not quite unique, since $K(p, q)=K\left(p, q^{-1}\right)$. For most knots (those with $q^{2} \not \equiv \pm 1 \bmod p$ ), there are two potential choices of normal form, which are related as follows. Suppose we start with the diagram for $K(p, q)$, which we think of as living in a plane. We can straighten out the overbridges by a plane isotopy, but at the cost of twisting up the underbridges. Flipping the resulting diagram over gives the normal form for $K\left(p, q^{-1}\right)$. 


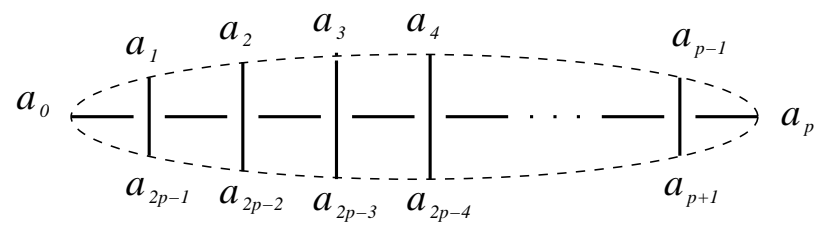

Figure 3: A neighborhood of an underbridge

\subsection{A Heegard splitting for $S^{3}-K(p, q)$}

The complement of a two-bridge knot admits a nice handle decomposition:

Proposition 2.2 $S^{3}-K(p, q)$ is the union of a genus two handlebody and a two-handle attached along a curve $\beta_{p, q}$. This curve is the boundary of a regular neighborhood of an overbridge in the Schubert normal form of $K(p, q)$.

Proof To obtain the desired decomposition, we start with the Schubert normal form of $K(p, q)$. The overbridges lie in a plane and the underbridges dip down below them. Split $S^{3}$ along this plane, and remove tubular neighborhoods of the underbridges. The part of $S^{3}$ which remains below the plane is a handlebody of genus 2. To get the part of $S^{3}-K(p, q)$ above the plane, we must separate the overbridges by adding a two-handle along $\beta_{p, q}$. Note that $\beta_{p, q}$ does not depend on which overbridge we choose, since the complement of a regular neighborhood of one overbridge is a regular neighborhood of the other.

A sample handle decomposition is shown in Figure 4, following the conventions of [6], Section 8. The region shown is part of the genus 2 surface $\Sigma_{2}$ which bounds the handlebody. The remainder of $\Sigma_{2}$ consists of two tubes below the plane of the picture, joining $A_{1}$ to $A_{2}$ and $B_{1}$ to $B_{2}$. The attaching circles of the handlebody are the two horizontal lines $\alpha_{1}$ and $\alpha_{2}$. The curve $\beta_{p, q}$ is shown in bold. It intersects each $\alpha_{i} p$ times.

\section{The generators of $\widehat{C F}\left(K^{n}\right)$}

From now on, we suppose that $K$ is a two-bridge knot and drop the $(p, q)$ when they are not relevant. We want to compute the various Ozsvath-Szabo Floer homologies for the three-manifold obtained by $n$ surgery on $K$, which we denote by $K^{n}$. Until the last section, we will consider only the case $n \gg 0$. 


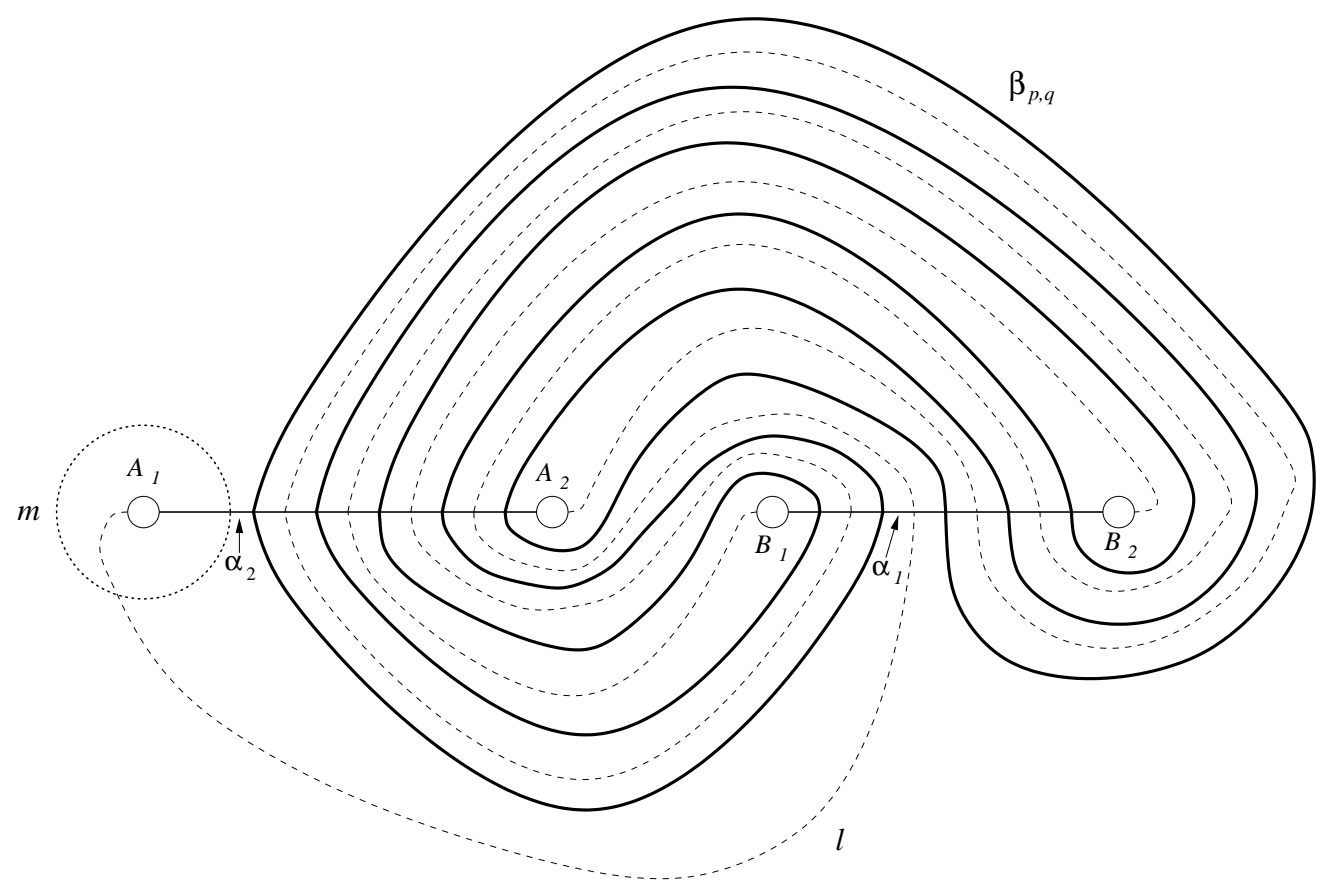

Figure 4: The Heegaard splitting for the complement of $K(5,3)$ (the figure-eight knot.) The longitude and meridian are shown by dashed lines. In a general knot, the longitude will spiral around the hole labeled $A_{1}$, so that it has intersection number zero with the class of $\alpha_{1}-\alpha_{2}$.

Our aim in this section is to write down generators for the complex $\widehat{C F}\left(K^{n}\right)$ and describe their relative gradings. Of course, these gradings will vary with the choice of $\operatorname{Spin}^{c}$ structure on $K^{n}$. There is a natural way to label these Spin $^{c}$ structures as $\mathbf{s}_{k}$, with respect to which we have the following result.

Proposition 3.1 For $n$ sufficiently large, $\widehat{C F}\left(K^{n}(p, q), \mathbf{s}_{k}\right)$ ( $q$ odd) admits a presentation with $p$ generators $x_{1}, x_{2}, \ldots x_{p}$. This presentation is independent of the value of $n$. For $k \geq g(K)$, the complexes $\widehat{C F}\left(K^{n}, \mathbf{s}_{k}\right)$ are all identical to a single complex which we refer to as $\widehat{C F}_{s}(K)$. The grading in this complex is given by

$$
\operatorname{gr}\left(x_{i+1}\right)-\operatorname{gr}\left(x_{i}\right)=(-1)^{\left\lfloor\frac{i q}{p}\right\rfloor}
$$

and there is a unique differential between $x_{2 i}$ and $x_{2 i+1}$. In general, the complex $\widehat{C F}\left(K^{n}, \mathbf{s}_{k}\right)$ is obtained by reflecting the complex $\widehat{C F}_{s}(K)$ at level $k$.

The process of reflection will be described in section 3.3. 


\subsection{The Heegaard splitting}

Our first order of business is to construct a Heegaard splitting for $K^{n}$. We start with the handlebody decomposition of $S^{3}-K\left(p, q^{-1}\right)$ described in Proposition 2.2. (The reason for our choice of $q^{-1}$ rather than $q$ will be apparent in 3.2.) When we do surgery, we attach a two-handle along a curve in the knot complement, and then fill in with a three-ball. Alternately, we can just think of attaching the two-handle to $\Sigma_{2}-\beta_{p, q}$. From this point of view, the homology classes of the longitude and meridian are represented by the curves $\ell$ and $m$ shown in Figure 4.

To do $n$-surgery, we attach the two-handle along the curve $\beta_{2}=\ell+n m$. The resulting three-manifold has a Heegaard diagram with attaching circles $\alpha_{1}, \alpha_{2}, \beta_{2}$, and $\beta_{1}=\beta_{p, q}$. An example is shown in Figure 5. There are several general features worth noting. First, the curve $\beta_{1}$ separates the four-times punctured sphere into two components: the exterior, which contains the point at infinity, and the interior. We orient the $\alpha_{i}$ so that they both point out of the exterior region and into the interior. Thus $\beta_{1}$ has the same intersection number with each of the $\alpha_{i}$. For $n \gg 0$, the curve $\beta_{2}$ always has a region with a large number of clockwise spirals, which we draw in the exterior.

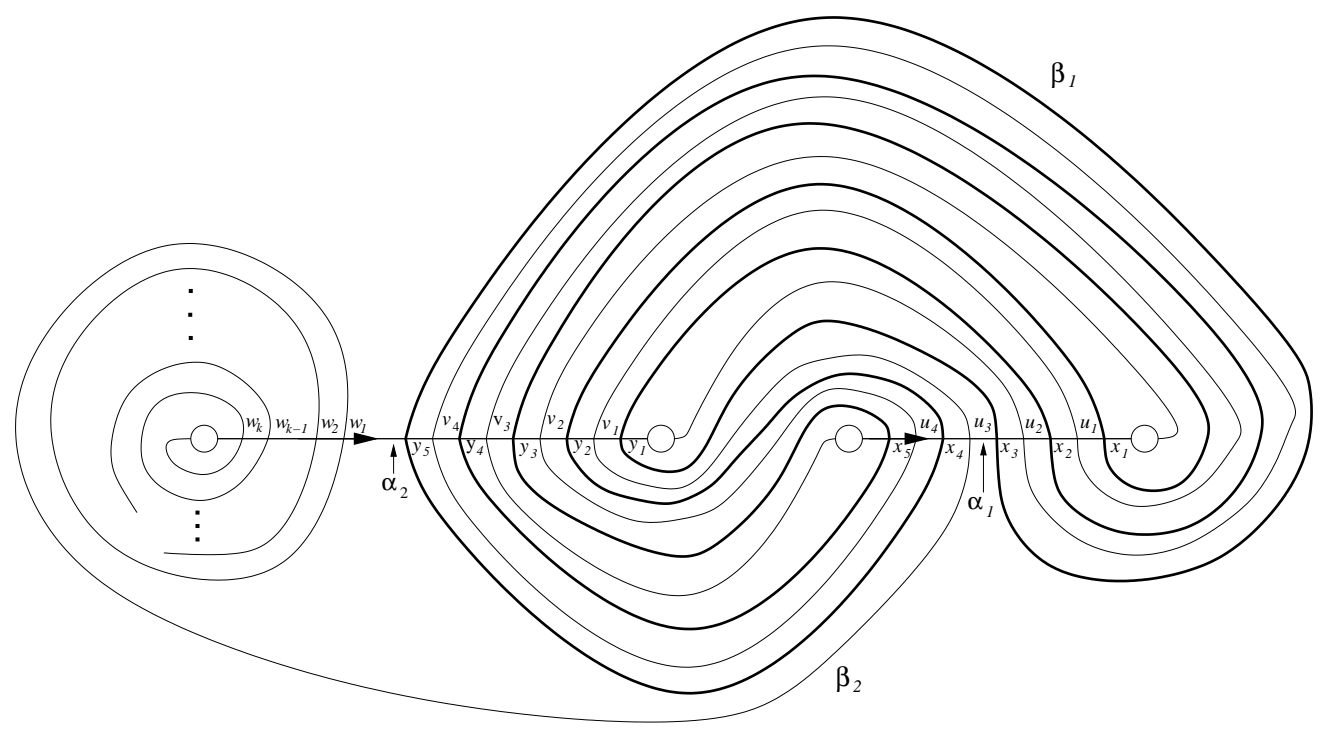

Figure 5: The Heegaard splitting for large $n$ surgery on $K(5,3)$. Note that for for large $n, \beta_{2}$ always has many clockwise spirals, although the exact number depends on the knot.

Recall that $\widehat{C F}$ is generated by the intersection points of the totally real tori

Algebraic 83 Geometric Topology, Volume 2 (2002) 
$T_{\alpha}, T_{\beta}$ in $s^{2} \Sigma_{2}$, or, equivalently, by unordered pairs of intersection points $\{x, y\}$ between the $\alpha_{i}$ and the $\beta_{i}$. We distinguish five kinds of such intersection points: between $\alpha_{1}$ and $\beta_{1}, \alpha_{2}$ and $\beta_{1}, \alpha_{1}$ and $\beta_{2}, \alpha_{2}$ and $\beta_{2}$ (not in the spiral), and $\alpha_{2}$ and $\beta_{2}$ (in the spiral). We label these points by $x_{i}, y_{j}, u_{k}, v_{l}, w_{m}$ respectively, as shown in Figure 5. (The convention is that the segment of $\alpha_{1}$ between $x_{1}$ and the hole lies in the interior region.) A priori, we are concerned with pairs of intersection points of the form $\left\{x_{i}, v_{l}\right\},\left\{x_{i}, w_{m}\right\}$ and $\left\{y_{j}, u_{k}\right\}$.

\section{$3.2 \quad \epsilon$-grading and basepoints}

Recall from [6] that there is an affine grading

$$
\epsilon: T_{\alpha} \cap T_{\beta} \rightarrow \text { Affine }\left(H_{1}\left(K^{n}\right)\right) \text {. }
$$

If we fix a basepoint $z \in \Sigma_{2}$, this grading corresponds to the usual grading of Seiberg-Witten Floer homology by $\operatorname{Spin}^{c}$ structures. Often, however, it is more convenient to fix an $\epsilon$-equivalence class and vary the basepoint to get all $\operatorname{Spin}^{c}$ structures. This is the approach we will take.

Our first observation is that we need only consider the pairs $\left\{x_{i}, w_{m}\right\}$ :

Lemma 3.1 For $n$ sufficiently large, there is an $\epsilon$-equivalence class which contains only points of the form $\left\{x_{i}, w_{m}\right\}$.

Proof There are $n$ equivalence classes, but the number of pairs $\left\{x_{i}, v_{l}\right\}$ and $\left\{y_{j}, u_{k}\right\}$ is bounded independent of $n$.

To describe $\epsilon$ explicitly, we need to discuss $H_{1}\left(K^{n}\right)$. We start with $H_{1}\left(\Sigma_{2}\right)$, which is a free abelian group generated by elements $A_{1}, A_{2}, B_{1}, B_{2}$, where $A_{i}$ is the class represented by the curve $\alpha_{i}$, and the $B_{i}$ link the "holes" in Figure 5 , so that $A_{i} \cdot B_{j}=\delta_{i j}$. We have

$$
\begin{aligned}
H_{1}\left(K^{n}\right) & \cong H_{1}\left(\Sigma_{2}\right) /\left\langle\alpha_{1}, \alpha_{2}, \beta_{1}, \beta_{2}\right\rangle \\
& \cong \mathbf{Z}\left\langle B_{1}\right\rangle \oplus \mathbf{Z}\left\langle B_{2}\right\rangle /\left\langle B_{1}+B_{2}, \beta_{2}\right\rangle \\
& \cong \mathbf{Z}\left\langle B_{2}\right\rangle /\left\langle n B_{2}\right\rangle .
\end{aligned}
$$

It follows that the quotient map $H_{1}\left(\Sigma_{2}\right) \rightarrow H_{1}\left(K^{n}\right)$ is given by

$$
x \rightarrow\left[x \cdot\left(A_{1}-A_{2}\right)\right] B_{2} .
$$

Lemma 3.2 There are affine gradings

$$
\begin{aligned}
\epsilon_{x} & :\left\{x_{i}\right\} \rightarrow \text { Affine }(\mathbf{Z}) \\
\epsilon_{w} & :\left\{w_{m}\right\} \rightarrow \text { Affine }(\mathbf{Z} / n)
\end{aligned}
$$

so that $\epsilon\left(\left\{x_{i}, w_{l}\right\}\right)-\epsilon\left(\left\{x_{j}, w_{m}\right\}\right)=\left(\left[\epsilon_{x}\left(x_{i}\right)-\epsilon_{x}\left(x_{j}\right)\right]+\left[\epsilon_{w}\left(w_{l}\right)-\epsilon_{w}\left(w_{m}\right)\right]\right) B_{2}$. 
Proof (Following Lemma 8.3 of [6].) To define $\epsilon_{x}\left(x_{i}\right)-\epsilon_{x}\left(x_{j}\right)$, join $x_{i}$ to $x_{j}$ by a path $a$ along $\alpha_{1}$ and a path $b$ along $\beta_{1}$. Then for any $l, \epsilon\left\{x_{i}, w_{l}\right\}-\epsilon\left\{x_{j}, w_{l}\right\}$ is the homology class of the path $a-b$, i.e. $\left[(a-b) \cdot\left(A_{1}-A_{2}\right)\right] B_{2}$. We set

$$
\epsilon_{x}\left(x_{i}\right)-\epsilon_{x}\left(x_{j}\right)=(a-b) \cdot\left(A_{1}-A_{2}\right) .
$$

Note that the right-hand side is independent of our choice of $a$ and $b$, since

$$
\alpha_{1} \cdot\left(A_{1}-A_{2}\right)=\beta_{1} \cdot\left(A_{1}-A_{2}\right)=0 .
$$

The definition of $\epsilon_{w}$ is analogous, but $\beta_{2} \cdot\left(A_{1}-A_{2}\right)=n$, so $\epsilon_{w}$ is only defined modulo $n$. The lemma now follows from the additivity of $\epsilon$.

Lemma $3.3 \epsilon_{w}\left(w_{m+1}\right)-\epsilon_{w}\left(w_{m}\right) \equiv 1 \bmod n$.

Proof This is obvious from Figure 5 .

Lemma $3.4 \epsilon_{x}\left(x_{i+1}\right)-\epsilon_{x}\left(x_{i}\right)=(-1)^{\left\lfloor\frac{i q}{p}\right\rfloor}$.

Proof Suppose that $i$ is even, so that the segment of $\alpha_{1}$ between $x_{i}$ and $x_{i+1}$ lies in the interior region, which is essentially a regular neighborhood of an overbridge. If we straighten this region out, we get a diagram that looks like Figure 6. We choose paths $a$ and $b$ as shown. All the contributions to the intersection number $(a-b) \cdot\left(A_{1}-A_{2}\right)$ cancel in pairs, with the exception of the single point marked with a star. Thus $\epsilon_{x}\left(x_{i+1}\right)-\epsilon_{x}\left(x_{i}\right)$ is +1 if $x_{i}$ is above $x_{i+1}$ in the diagram, and -1 otherwise. To see which case holds, recall from section 2 that straightening out the overbridges in the normal form for $K\left(p, q^{-1}\right)$ gives us the normal form for $K(p, q)$, with $\alpha_{1}$ playing the role of an overbridge $O_{1}$. The segment of $\alpha_{1}$ between $x_{i}$ and $x_{i+1}$ corresponds to $O_{1}$ 's $i$ th intersection with an underbridge. If we label the intersection points on $\beta_{1}$ as in Figure 3, $x_{i}$ will correspond to the point $a_{i q}$, which is in the top half of the diagram if and only if $0<i q<p \bmod 2 p$. This proves the result when $i$ is even. When $i$ is odd, we argue similarly using the exterior region.

Using the formula of Lemma 3.4, it is not difficult to see that

$$
\epsilon_{x}\left(x_{i+1}\right)-\epsilon_{x}\left(x_{i}\right)=\epsilon_{x}\left(x_{p+1-i}\right)-\epsilon_{x}\left(x_{p-i}\right) .
$$

It follows that $\epsilon_{x}$ is symmetric under the involution $\iota$ which sends $x_{i} \mapsto x_{p+1-i}$, in the sense that

$$
\epsilon_{x}\left(x_{i}\right)-\epsilon_{x}\left(x_{j}\right)=-\left[\epsilon_{x}\left(\iota\left(x_{i}\right)\right)-\epsilon_{x}\left(\iota\left(x_{j}\right)\right)\right] .
$$




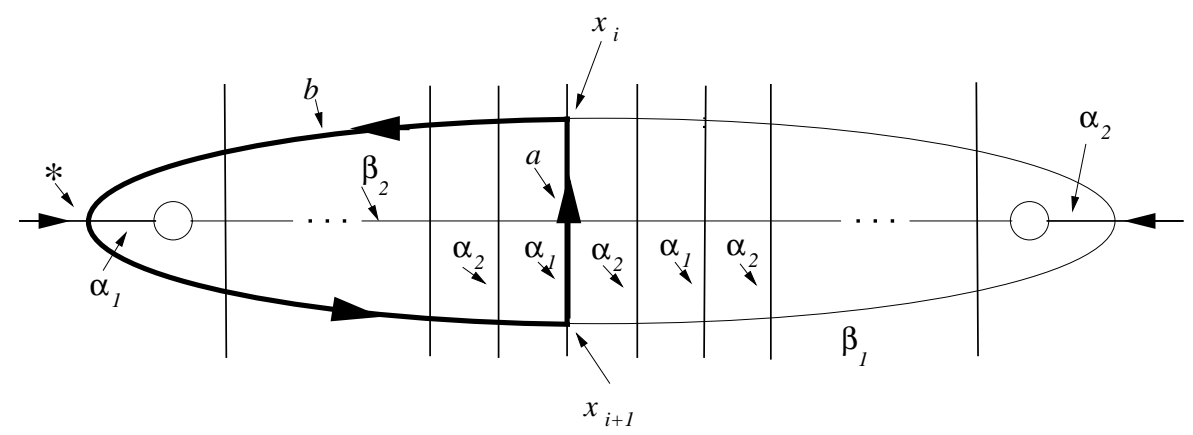

Figure 6: The interior region. We have drawn the case when $x_{i}$ is above $x_{i+1}$, but the reverse may also be true.

As a result, there is a natural lift of $\epsilon_{x}$ to a $\mathbf{Z}$ valued map (rather than just Affine $(\mathbf{Z})$ valued), namely the one which has $\epsilon_{x}\left(x_{i}\right)=-\epsilon_{x}\left(\iota\left(x_{i}\right)\right)$. We call this lift the Alexander grading on the $x_{i}$. (The name is explained by Proposition 3.5.)

Putting Lemmas 3.1 to 3.3 together, we get the following corollary, which is an exact analogue of Proposition 8.3 of [6].

Corollary 3.1 For $n$ sufficiently large and an appropriate choice of $M$, there is an $\epsilon$-equivalence class containing only the pairs $\left\{x_{i}, w_{M-\epsilon_{x}\left(x_{i}\right)}\right\}$.

From now on, we choose one such equivalence class and work with it. In this case, we can drop the $w$ 's and refer to the generators as $x_{i}$ without ambiguity. To get different $\operatorname{Spin}^{c}$ structures, we vary the basepoint. As in [6], we consider choices of basepoint which lie inside the spiral region. Denote by $z_{i}$ a point lying in the rectangular region with corners at $w_{i+1}, w_{i}, w_{i-1}$, and $w_{i}$ again. (See Figure 7). We will call the $\operatorname{Spin}^{c}$ structure determined by the basepoint $z_{M-k}$ and our fixed $\epsilon$-equivalence class $\mathbf{s}_{k}$. It follows from Lemma 2.12 of [6] that $\mathbf{s}_{k}$ is independent of which $\epsilon$-equivalence class we took. Although the $\mathbf{s}_{k}$ may not represent every $\operatorname{Spin}^{c}$ structure on $K^{n}$, we we will see that they include all of the interesting ones.

\subsection{Annular differentials}

In this section, we describe certain differentials in the chain complex $C F^{+}$. As a corollary, we obtain the grading in $\widehat{C F}\left(K^{n}, \mathbf{s}_{k}\right)$ for every $\mathbf{s}_{k}$. The key tool is the following proposition. 
Proposition 3.2 (Lemma 8.4 of [6]) Suppose we are given $\mathbf{x}, \mathbf{y} \in T_{\alpha} \cap T_{\beta} \subset$ $s^{2} \Sigma_{2}$, and an element $\phi \in \pi_{2}(\mathbf{x}, \mathbf{y})$ whose domain is an annulus with one $270^{\circ}$ corner. Then $\mu(\mathbf{x}, \mathbf{y})=1$ and the class $\phi$ has a unique holomorphic representative.

We refer to such a class as an annular differential from $\mathbf{x}$ to $\mathbf{y}$, or, if we wish to allow either $\phi \in \pi_{2}(\mathbf{x}, \mathbf{y})$ or $\phi \in \pi_{2}(\mathbf{y}, \mathbf{x})$, as an annular differential between $\mathbf{x}$ and $\mathbf{y}$. This definition is useful to us because there is an obvious annular differential $\phi_{i}$ between $x_{i}$ and $x_{i+1}$. Its domain can roughly be described as the region bounded by the curves $a$ and $b$ in Figure 6. More precisely, if $i$ is even, the segment of $\alpha_{1}$ joining $x_{i}$ to $x_{i+1}$ divides the interior region into 2 components. The domain of $\phi_{i}$ consists of one of these components, together with a portion of the spiral like that shown in Figure 7 and the tube joining them. We call $\phi_{i}$ an interior differential. Similarly, if $i$ is odd, the segment of $\alpha_{1}$ joining $x_{i}$ to $x_{i+1}$ divides the exterior region into 2 components. The domain of the exterior differential $\phi_{i}$ is the one which contains the spiral, but with the shaded region of Figure 7 removed.

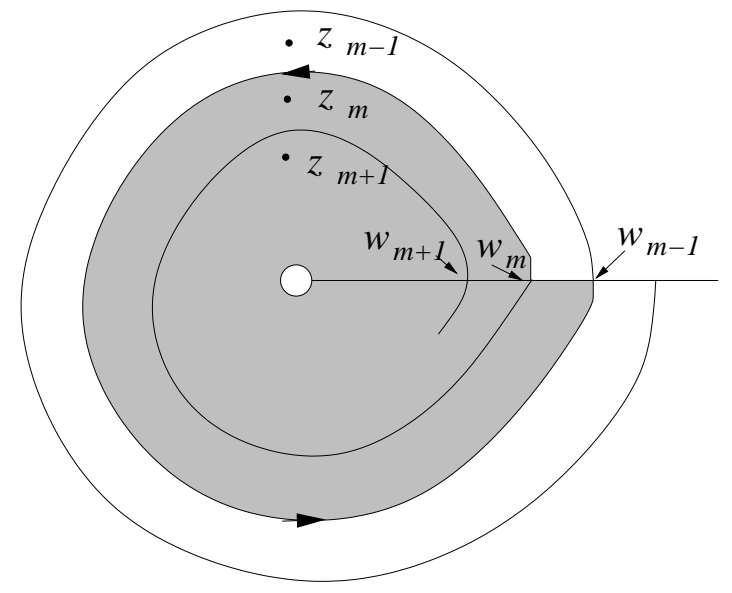

Figure 7: A neighborhood of the spiral. The shaded region is part of the domain of an interior differential from $\left\{x_{i}, w_{m-1}\right\}$ to $\left\{x_{j}, w_{m}\right\}$.

To describe how the annular differentials fit into the complex $\widehat{C F}$, we need to fix a $\operatorname{Spin}^{c}$ structure. Initially, we choose one whose associated basepoint is in the outer part of the spiral. More specifically, let $G=\max _{i}\left\{\epsilon_{x}\left(x_{i}\right)\right\}$. (We will show in 3.4 that $G=g(K)$.) Then if $k \geq G$, the point $z_{M-k}$ is further out in the spiral than all of the $w_{M-\epsilon_{x}\left(x_{i}\right)}$. 
Proposition 3.3 If $k \geq G, \operatorname{gr}\left(x_{i}\right)=\epsilon_{x}\left(x_{i}\right)$ in $\widehat{C F}\left(K^{n}, \mathbf{s}_{k}\right)$. There is a unique differential between $x_{2 j}$ and $x_{2 j+1}$.

Proof To check the statement about the grading, it suffices to show that

$$
\operatorname{gr}\left(x_{i+1}\right)-\operatorname{gr}\left(x_{i}\right)=\epsilon_{x}\left(x_{i+1}\right)-\epsilon_{x}\left(x_{i}\right) .
$$

Suppose $i$ is even, so that we have an interior differential $\phi_{i}$ between $x_{i}$ and $x_{i+1}$. Now since $k \geq G$, we have $n_{z_{M-k}}\left(\phi_{i}\right)=0$, so $\operatorname{gr}\left(x_{i+1}\right)-\operatorname{gr}\left(x_{i}\right)=$ $\pm \mu\left(\phi_{i}\right)= \pm 1$, depending on whether $\phi_{i}$ is a differential from $x_{i+1}$ to $x_{i}$ or the other way around. To tell, we give the boundary of the domain of $\phi_{i}$ its standard orientation and see which way the $\alpha$ segments point. Looking at Figure 7, we see that the differential goes from the point with larger $\epsilon_{x}$ to the one with smaller $\epsilon_{x}$. This proves the claim.

For $i$ odd, the argument is similar, except that we now have $n_{z_{M-k}}\left(\phi_{i}\right)=1$, and the boundary circle in Figure 7 is oriented clockwise. Thus the differential goes to the point with larger $\epsilon_{x}$. Without loss of generality, we assume $\epsilon_{x}\left(x_{i+1}\right)<$ $\epsilon_{x}\left(x_{i}\right)$ and compute

$$
\begin{aligned}
\operatorname{gr}\left(x_{i+1}\right)-\operatorname{gr}\left(x_{i}\right) & =\mu\left(\phi_{i}\right)-2 n_{z_{M-k}}\left(\phi_{i}\right) \\
& =1-2 \\
& =\epsilon_{x}\left(x_{i+1}\right)-\epsilon_{x}\left(x_{i}\right) .
\end{aligned}
$$

To get the last statement, note that $n_{x_{M-k}}\left(\phi_{2 j}\right)=0$, so the interior differential is "turned on" in $\widehat{C F}\left(K^{n}, \mathbf{s}_{k}\right)$. By Proposition 3.2, there is a unique element in $\mathcal{M}\left(\phi_{2 j}\right)$.

We now know that the complexes $\widehat{C F}\left(K^{n}, \mathbf{s}_{k}\right)$ have the same generators and grading for $k \geq G$. We wish to show that they have the same differentials as well. To do so, we use the following result.

Lemma 3.5 Assume $n \gg G$, and suppose $\phi \in \pi_{2}\left(x_{i}, x_{j}\right)$ has $\mu(\phi)=1$. Then one boundary component of $\mathcal{D}(\phi)$ consists of the segments of $\alpha_{1}$ and $\beta_{2}$ which join $w_{M-\epsilon_{x}\left(x_{i}\right)}$ and $w_{M-\epsilon_{x}\left(x_{j}\right)}$ and which lie inside the spiral.

Proof Since $\mu(\phi)=1$, we have

$$
n_{z_{M-k}}(\phi)=\left[\operatorname{gr}\left(x_{i}\right)-\operatorname{gr}\left(x_{j}\right)-1\right] / 2
$$

where the grading is taken with respect to the complex $\widehat{H F}\left(K^{n}, \mathbf{s}_{k}\right)$. Since the gradings are constant for $k \geq G, n_{z_{M-k}}(\phi)$ is constant as well. But this can only happen if $\partial \mathcal{D}(\phi)$ is disjoint from the outer part of the spiral, and thus from the inner part too. Given this, it is easy to see that $\partial \mathcal{D}(\phi)$ must have a component as described. 
a)
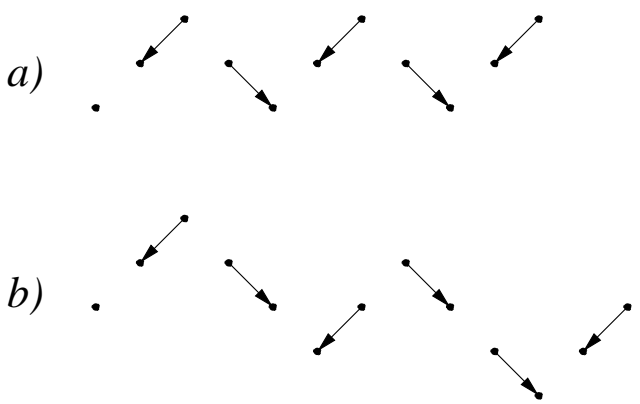

Figure 8: The stable complexes of (a) $K(11,5)$ and (b) $K(13,5)$. The generators $x_{1}, x_{2}, \ldots, x_{p}$ are represented by dots, running from left to right. The grading of each generator is shown by its height, and interior differentials are indicated by arrows.

Thus for any differential $\phi$, we have $n_{z_{k}}(\phi)=n_{z_{l}}(\phi)$ if $k, l \geq G$. It follows that for $n$ sufficiently large and $k \geq G$, the complexes $\widehat{C F}\left(K^{n}, \mathbf{s}_{k}\right)$ are all isomorphic to a single complex, which we call the stable complex of $K^{n}$ (written $\widehat{C F}_{s}\left(K^{n}\right)$.) Although its cohomology is basically trivial, $\widehat{C F}_{s}\left(K^{n}\right)$ turns out to be a very useful object. In particular, if we combine Lemma 3.4 with Proposition 3.3, we see that the grading in $\widehat{C F}_{s}\left(K^{n}\right)$ is very easy to compute. A few examples of stable complexes are shown in Figure 8. The reader is encouraged to draw some others and familiarize himself with their behavior.

Warning Like the Schubert normal form, the stable complex is not quite canonical: thinking of $K(p, q)$ as $K\left(p, q^{-1}\right)$ gives a different complex.

We now consider what happens to the complex $\widehat{C F}$ if we choose a $\operatorname{Spin}^{c}$ structure $\mathbf{s}_{k}$ with $k<G$. We still have the same interior and exterior differentials $\phi_{i}$, but the value of $n_{z_{M-k}}\left(\phi_{i}\right)$ will change. More precisely, suppose that $\phi$ is an interior differential from $x_{i}$ to $x_{j}$. Looking at Figure 7 , we see that we have

$$
n_{z_{k}}(\phi)= \begin{cases}0 & \text { if } k<M-\epsilon_{x}\left(x_{j}\right) \\ 1 & \text { if } k>M-\epsilon_{x}\left(x_{i}\right) .\end{cases}
$$

Likewise, if $\phi$ is an exterior differential from $x_{i}$ to $x_{j}$, we have

$$
n_{z_{k}}(\phi)= \begin{cases}1 & \text { if } k<M-\epsilon_{x}\left(x_{j}\right) \\ 0 & \text { if } k>M-\epsilon_{x}\left(x_{i}\right) .\end{cases}
$$

Applying this to our differentials $\phi_{i}$, we obtain:

Algebraic 63 Geometric Topology, Volume 2 (2002) 
Proposition 3.4 In $\widehat{C F}\left(K^{n}, \mathbf{s}_{k}\right)$, we have the following proposition.

$$
\operatorname{gr}\left(x_{i+1}\right)-\operatorname{gr}\left(x_{i}\right)= \begin{cases}\epsilon_{x}\left(x_{i+1}\right)-\epsilon_{x}\left(x_{i}\right) & \text { if } \min \left\{\epsilon_{x}\left(x_{i}\right), \epsilon_{x}\left(x_{i+1}\right)\right\}<k \\ -\left[\epsilon_{x}\left(x_{i+1}\right)-\epsilon_{x}\left(x_{i}\right)\right] & \text { if } \min \left\{\epsilon_{x}\left(x_{i}\right), \epsilon_{x}\left(x_{i+1}\right)\right\} \geq k .\end{cases}
$$

Proof If $\min \left\{\epsilon_{x}\left(x_{i}\right), \epsilon_{x}\left(x_{i+1}\right)\right\}<k$, then the value of $n_{z_{M-k}}\left(\phi_{i}\right)$ is the same as in the stable case, so the difference in gradings is the same as well. Otherwise, it is the reverse of the stable case ( 0 for an exterior differential, 1 for an interior), and we argue as in the proof of Proposition 3.3.

The content of this proposition is best understood pictorially: to obtain $\widehat{C F}\left(K^{n}, \mathbf{s}_{k}\right)$, we start with the stable complex and reflect all the points that lie above level $k$, as illustrated in Figure 9. Note that in the reflected portion the exterior differentials have been "turned on" and the interior differentials are "turned off".

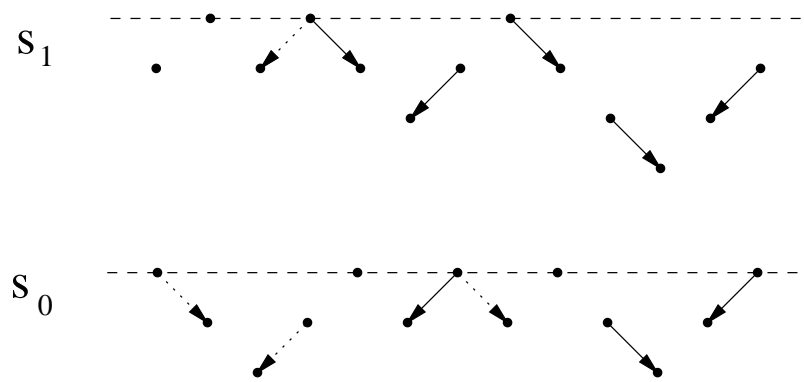

Figure 9: $\widehat{C F}\left(K(13,5), \mathbf{s}_{1}\right)$ and $\widehat{C F}\left(K(13,5), \mathbf{s}_{0}\right)$. Exterior differentials are shown by dashed lines. In each case, the line of reflection is drawn in. (Compare with Figure 8b.)

At this point, we have more or less completed the proof of Proposition 3.1. The only thing which remains to be proved is the statement that $\widehat{C F}\left(K^{n}, \mathbf{s}_{k}\right)$ is independent of $n$ for $n$ sufficiently large. To see this, we use Lemma 3.5 again. Indeed, the lemma implies that the domains of potential differentials are independent of $n$. From this, it is not difficult to conclude that the differentials themselves are independent of $n$, and thus that the complexes $\widehat{C F}\left(K^{n}, \mathbf{s}_{k}\right)$ and $C F^{ \pm}\left(K^{n}, \mathbf{s}_{k}\right)$ are as well. When $n \gg 0$, we are thus justified in dropping it from our notation and simply writing $\widehat{C F}\left(K, \mathbf{s}_{k}\right)$ and $C F^{ \pm}\left(K, \mathbf{s}_{k}\right)$.

\subsection{Knot invariants and the stable complex}

We end this section by describing some relations between $\widehat{C F}_{s}(K)$ and classical knot invariants. Unsurprisingly, there is a connection with the Alexander 
polynomial:

Proposition 3.5 Let $n_{k}$ denote the number of $x_{i}$ in the stable complex of $K$ with $\epsilon_{x}\left(x_{i}\right)=k$. Then the normalized, symmetrized Alexander polynomial of $K$ is given by

$$
\Delta_{K}(t)=(-1)^{\epsilon_{x}\left(x_{1}\right)} \sum_{k} n_{k}(-t)^{k}
$$

Proof From the Heegard splitting of section 2.1, we see that $\pi_{1}\left(S^{3}-K\right)$ has a presentation $\left\langle a_{1}, a_{2} \mid w_{\beta_{1}}\right\rangle$, where $w_{\beta_{1}}$ is the image of $\beta_{1}$ in $\pi_{1}\left(H_{1}\right)=\left\langle a_{1}, a_{2}\right\rangle$. $w_{\beta_{1}}$ admits the following concrete description: let $w$ be the empty word. Start anywhere on $\beta_{1}$ and travel along it in either direction. Every time you cross $\alpha_{1}$, append $a_{1}^{ \pm 1}$ to the end of $w\left(+1\right.$ if you go up through $\alpha_{1}$ as determined by the orientation, -1 if you go down.) Similarly, append $a_{2}^{ \pm 1}$ to $w$ every time you pass through $\alpha_{2}$. When you get back your starting point, $w$ will be $w_{\beta_{1}}$. We use the orientation convention of section 3.1, according to which the $\alpha_{i}$ are oriented so that $\left[a_{1}\right]=-\left[a_{2}\right]=t$ in $H_{1}\left(S^{3}-K\right)$, but any choice of orientations would work just as well.

We now observe that the Alexander grading is just a geometrical interpretation of the free differential, so that

$$
\sum(-t)^{\epsilon_{x}\left(x_{i}\right)}= \pm t^{k} d_{a_{1}} w_{\beta_{1}}
$$

for some $k$. Indeed, each term in $d_{a_{1}} w_{\beta_{1}}$ corresponds to an intersection point of $\alpha_{1}$ and $\beta_{1}$. If $u$ and $v$ are two such points, we claim that $\epsilon_{x}(u)-\epsilon_{x}(v)$ is the difference between the corresponding exponents of $t$ in $d_{a_{1}} w_{\beta_{1}}$. To see this, recall that $\epsilon_{x}(u)-\epsilon_{x}(v)$ is the homology class in $H_{1}\left(S^{3}-K\right)$ of a loop $\gamma$ which goes from $u$ to $v$ along $\beta_{1}$, and then from $v$ to $u$ along $\alpha_{1}$. The class of $\gamma$ can be determined by counting (with sign) its intersections with $\alpha_{1}$ and subtracting off its intersections with $\alpha_{2}$. The difference in the exponents of $t$ in $d_{a_{1}} w_{\beta_{1}}$ counts exactly the same quantity. To check that the signs agree, note that the sign of a term in the free differential is the sign of the associated intersection point $u$, which is $(-1)^{\operatorname{gr}(u)}=(-1)^{\epsilon_{x}(u)}$.

Strictly speaking, the Alexander polynomial is the gcd of $d_{a_{1}} w_{\beta_{1}}$ and $d_{a_{2}} w_{\beta_{1}}$, but an argument of McMullen (Theorem 5.1 of [4]) shows that when the presentation of $\pi_{1}$ comes from a Heegard splitting, $d_{a_{1}} w_{\beta_{1}}$ and $d_{a_{2}} w_{\beta_{1}}$ agree up to a unit. Thus

$$
\sum(-t)^{\epsilon_{x}\left(x_{i}\right)}
$$


represents $\Delta_{K}(t)$. It is symmetric, so it must be the symmetrized Alexander polynomial. Finally, it is easy to check that our choice of the sign $(-1)^{\epsilon_{x}\left(x_{1}\right)}$ normalizes $\Delta_{K}$ to have $\Delta_{K}(1)=1$.

Corollary 3.2 The genus of $K$ is the maximum value assumed by $\epsilon_{x}$.

Proof All two-bridge knots are alternating. For such knots, it is well-known that the genus is the highest power of $t$ appearing in the symmetrized Alexander polynomial.

Interestingly, there is also a relationship with the signature.

Proposition $3.6 \quad \sigma(K)=\epsilon_{x}\left(x_{1}\right)-\epsilon_{x}\left(x_{p}\right)$

Proof This is a restatement of Theorem 9.3.6 of [5].

\section{Computing the homology}

We now turn to our main task, which is to compute the homology of $\widehat{C F}\left(K, \mathbf{s}_{k}\right)$ and $C F^{ \pm}\left(K, \mathbf{s}_{k}\right)$. As described in the introduction, the Floer homology of the zero surgery splits naturally into two parts: a group $V$ which looks like the Floer homology for a torus knot of the same signature, and a group $Q$ which is concentrated in a single grading. In $\widehat{C F}\left(K, \mathbf{s}_{k}\right)$, there will be a third summand $\mathbf{Z}\left[u^{-1}\right]$. If $\sigma(K)>0, V$ will be absorbed into the $\mathbf{Z}\left[u^{-1}\right]$ summand, while if $\sigma(K)<0$, it will not.

To describe $Q$ and $V$ precisely, we need some notation. Let $a_{k}$ be the coefficient of $t^{k}$ in the symmetrized Alexander polynomial of $K$, and set

$$
u_{k}=\sum_{i>k}(i-k) a_{i}
$$

$u_{k}$ is a familiar quantity: it is just the Seiberg-Witten invariant associated to the $k$ th $\operatorname{Spin}^{c}$ structure on $K^{0}$. We let $\sigma$ be the signature of the two-bridge knot $K$, and set

$$
\begin{aligned}
\sigma^{\prime} & =\sigma / 2 \\
h_{k} & =\max \left(\left\lceil\frac{\left|\sigma^{\prime}\right|-k}{2}\right\rceil, 0\right) \\
b_{k} & =u_{k}+(-1)^{k-\sigma^{\prime}} h_{k} .
\end{aligned}
$$

Then we have the following: 
Theorem 4.1 For $k \geq 0$, there is an isomorphism of $\mathbf{Z}[u]$ modules

$$
H F^{+}\left(K, \mathbf{s}_{k}\right) \cong H F^{+}\left(K, \mathbf{s}_{-k}\right) \cong \begin{cases}Q_{k} \oplus \mathbf{Z}\left[u^{-1}\right] & \text { if } \sigma \geq 0 \\ Q_{k} \oplus V_{k} \oplus \mathbf{Z}\left[u^{-1}\right] & \text { if } \sigma \leq 0\end{cases}
$$

where $Q_{k} \cong \mathbf{Z}^{\left|b_{k}\right|}$ and $V_{k} \cong \mathbf{Z}\left[u^{-1}\right] / u^{-h_{k}}$. All the elements of $Q_{k}$ have grading $k-1$, and $1 \in V_{k}$ has grading $k-2 h_{k}$ if $k \equiv \sigma^{\prime} \bmod 2$ and $k+1-2 h_{k}$ otherwise. Finally, $1 \in \mathbf{Z}\left[u^{-1}\right]$ has grading $\sigma^{\prime}$ if $\sigma \leq 0$, or $\sigma^{\prime}-2 h_{k}$ if $\sigma \geq 0$.

Warning For ease of expression, we have chosen to state the gradings in absolute terms, although they are only meaningful relative to each other. They do not correspond to the absolute gradings discussed in [8].

Corollary 4.1

$$
\widehat{H F}\left(K, \mathbf{s}_{k}\right) \cong \begin{cases}\widehat{Q_{k}} \oplus \mathbf{Z} & \text { if } \sigma \geq 0 \\ \widehat{Q_{k}} \oplus \widehat{V_{k}} \oplus \mathbf{Z} & \text { if } \sigma \leq 0\end{cases}
$$

where $\widehat{Q_{k}} \cong Q_{k} \otimes H^{*}\left(S^{1}\right)$ and $\widehat{V_{k}} \cong H^{*}\left(S^{2 h_{k}-1}\right)$. The grading of $1 \in H^{*}\left(S^{2 h_{k}-1}\right)$ is the same as that of $1 \in V_{k}$, and the grading of $1 \in \mathbf{Z}$ is the same as that of $1 \in \mathbf{Z}\left[u^{-1}\right]$.

\section{Corollary 4.2}

$$
H F^{-}\left(K^{n}, \mathbf{s}_{k}\right) \cong \begin{cases}Q_{k} \oplus \mathbf{Z}[u] & \text { if } \sigma \geq 0 \\ Q_{k} \oplus V_{k} \oplus \mathbf{Z}[u] & \text { if } \sigma \leq 0\end{cases}
$$

The gradings are shifted from those in $\mathrm{HF}^{+}$. More precisely, the grading of $1 \in \mathbf{Z}[u]$ is 2 less than that of $1 \in \mathbf{Z}\left[u^{-1}\right] \subset H F^{+}\left(K^{n}, \mathbf{s}_{k}\right)$, while the gradings of $V_{k}$ and $Q_{k}$ are 1 less than those of their counterparts in $H F^{+}\left(K^{n}, \mathbf{s}_{k}\right)$.

\section{1 $C F^{\infty}(K)$ and $C F^{ \pm}(K)$}

Until now, we have only discussed the complex $\widehat{C F}(K)$. We will need to use $C F^{\infty}(K), C F^{+}(K)$, and $C F^{-}(K)$ as well, so we collect some basic facts about them from [6], [7] here.

We begin with $C F^{\infty}$. Visually, this complex may be obtained by stacking copies of $\widehat{C F}$ together, each two units apart from the next. The stack extends infinitely in both directions. As observed in Theorem 8.9 of [6], the complex we get is independent of our choice of Spin ${ }^{c}$ structure. Depending on the situation, we will use one of two different conventions to label the generators of $C F^{\infty}$. If 
we have fixed a $\operatorname{Spin}^{c}$ structure $\mathbf{s}$, we follow the notation of [6] and consider the generators as pairs $[\mathbf{x}, j]$. On the other hand, if we want to avoid specifying a $\operatorname{Spin}^{c}$ structure, it is often convenient to choose a zero level for the grading on $C F^{\infty}$ and let $(\mathbf{x}, j)$ denote the generator above $\mathbf{x}$ with grading $j$.

For any choice of $\operatorname{Spin}^{c}$ structure $\mathbf{s}, C F^{\infty}(M)$ has a filtration by subcomplexes $\ldots \subset F_{-1}^{\mathbf{s}} \subset F_{0}^{\mathbf{s}} \subset F_{1}^{\mathbf{s}} \subset \ldots$, where $F_{i}^{\mathbf{s}}=\{[\mathbf{x}, j] \mid j<i\}$. For each $i$, we have

$$
\begin{aligned}
C F^{-}(M, \mathbf{s}) & \cong F_{i}^{\mathbf{s}} \\
C F^{+}(M, \mathbf{s}) \cong C F^{\infty}(M) / F_{i}^{\mathbf{s}} & \widehat{C F}(M, \mathbf{s}) \cong F_{i}^{\mathbf{s}} / F_{i-1}^{\mathbf{s}} .
\end{aligned}
$$

Frequently, we wish to relate $H F^{ \pm}$to $\widehat{H F}$. In one direction, this is accomplished by the following Gysin sequence:

Lemma 4.1 There is a long exact sequence:

$$
\longrightarrow \widehat{H F}_{i}(M, \mathbf{s}) \longrightarrow H F_{i}^{+}(M, \mathbf{s}) \stackrel{u}{\longrightarrow} H F_{i-2}^{+}(M, \mathbf{s}) \longrightarrow \widehat{H F}_{i-1}(M, \mathbf{s})
$$

Proof At the chain level, the map $u: C F^{+}(M, \mathbf{s}) \rightarrow C F^{+}(M, \mathbf{s})$ is clearly a surjection with kernel $\widehat{C F}(M, \mathbf{s})$. Thus we have a short exact sequence

$$
0 \longrightarrow \widehat{C F}_{*}(M, \mathbf{s}) \longrightarrow C F_{*}^{+}(M, \mathbf{s}) \stackrel{u}{\longrightarrow} C F_{*-2}^{+}(M, \mathbf{s}) \longrightarrow 0
$$

Conversely, to go from $\widehat{H F}$ to $H F^{+}$, we can sometimes use the following:

Lemma 4.2 There is a spectral sequence with $E_{2}$ term $\widehat{H F}(M, \mathbf{s}) \otimes \mathbf{Z}\left[u^{-1}\right]$ which converges to $\mathrm{HF}^{+}(\mathrm{M}, \mathbf{s})$.

Proof We have a filtration $\bar{F}_{0}^{\mathrm{s}} \subset \bar{F}_{1}^{\mathrm{s}} \subset \ldots$ of $C F^{+}(M, \mathbf{s})$, where $\bar{F}_{i}^{\mathrm{s}}$ is the

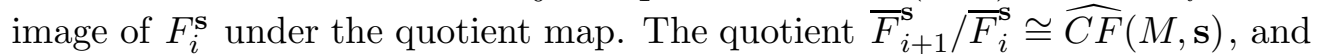
the usual arguments show that the first differential is just the differential in $\widehat{C F}$.

\subsection{Conjugation symmetry and the antistable complex}

It is well known that there is a conjugation symmetry

$$
H F^{ \pm}\left(K, \mathbf{s}_{k}\right) \cong H F^{ \pm}\left(K, \mathbf{s}_{-k}\right) .
$$

In our case, this symmetry is explicitly realized on the chain level by the map $\iota$ described in section 3.2. To see this, we observe that there is an exact symmetry between the interior and exterior regions of our Heegard diagram. 
Lemma 4.3 There is an involution $j: \Sigma_{2} \rightarrow \Sigma_{2}$ which preserves the $\alpha_{i}$ and the $\beta_{i} . j$ reverses the interior and exterior regions, and $j\left(x_{i}\right)=x_{p+1-i}$.

Proof We begin by defining $j$ on the punctured $S^{2}$ of our usual diagram. The circle $\beta_{1}$ divides $S^{2}-4 D^{2}$ into the interior and exterior regions, each of which is homeomorphic to the punctured disk of Figures 6 and 3. To obtain $S^{2}-4 D^{2}$, we identify 2 copies of this disk along their boundary by an orientation reversing diffeomorphism which sends the point $a_{i}$ to $a_{q-i}$. Let $j$ be the map which interchanges the two copies. Since $i \rightarrow q-i$ is an involution, $j$ respects the gluing, and thus defines an orientation preserving involution of $S^{2}-4 D^{2}$. It is easy to see that $j$ preserves $\alpha_{1}, \alpha_{2}$ and $\beta_{1}$, and it would preserve $\beta_{2}$ if there we no spiral. We solve this problem by pushing the spiral onto the adjacent tube, and extend $j$ to $\Sigma_{2}$ by an orientation preserving involution which switches the ends of the tubes and is chosen to preserve the spiral. Since $j$ reverses the ends of $\alpha_{1}$ in $S^{2}-4 D^{2}$, it must send $x_{i}$ to $x_{p+1-i}$.

It follows that $\iota$ induces an isomorphism $C F^{ \pm}\left(K, \mathbf{s}_{k}\right) \cong C F^{ \pm}\left(K, \mathbf{s}_{-k}\right)$. (Note, however, that we have made different choices of complex structure, etc. on the two sides of the equation. Since we are mostly interested in the homology, this will not be an issue.) When $k \ll 0$, the complex $C F^{ \pm}\left(K, \mathbf{s}_{k}\right)$ is independent of $k$, and we refer to it as the antistable complex $C F_{a}^{ \pm}(K)$ of $K$. It is obvious from the discussion above that $H F_{a}^{ \pm}(K) \cong H F^{ \pm}{ }_{s}(K)$.

\subsection{The homology of the stable complex}

We need one more result before we can work out $\mathrm{HF}^{+}\left(K^{n}\right)$ :

Proposition 4.1 The homology of $\widehat{C F}_{s}(K)$ is a single copy of $\mathbf{Z}$ with the same grading as $x_{1}$.

At first glance, this seems like a very modest statement. In fact, it follows easily from the general results of [7] that the homology of the stable complex must be $\mathbf{Z}$, so only real content of the proposition is to tell us the grading of the generator. Nonetheless, this result is a key step in the calculation. In particular, it is the only place at which the differentials make an appearance.

As is usual with Floer homology, the proof of Proposition 4.1 should be thought of in terms of Morse theory. For $\widehat{H F}$, the difference in the value of the "Morse function" between two critical points is given by the area of the Whitney disk joining them, or equivalently by the area of the associated domain in $\Sigma_{2}$. 
Consider the generators $x_{2 i}$ and $x_{2 i+1}$ in $\widehat{C F}_{s}(K)$. By Proposition 3.3, we know that there is an interior differential, which goes either from $x_{2 i}$ to $x_{2 i+1}$ or from $x_{2 i+1}$ to $x_{2 i}$. To avoid repeatedly stating both possibilities, we introduce alternate names $\hat{x}_{2 i}$ and $\hat{x}_{2 i+1}$ for $x_{2 i}$ and $x_{2 i+1}$, arranged so that the differential is always from $\hat{x}_{2 i}$ to $\hat{x}_{2 i+1}$.

Suppose we choose the metric on $\Sigma_{2}$ so that the interior region is very thin,and thus that the drop in the value of the "Morse function" between $\hat{x}_{2 i}$ and $\hat{x}_{2 i+1}$ is quite small. Now if we were really doing Morse theory, the logical conclusion would be that $\hat{x}_{2 i}$ and $\hat{x}_{2 i+1}$ are a pair of critical points created by some small perturbation, and we should just cancel them. If we could do this to all the pairs, we would be left with nothing but $x_{1}$, and the result would be obvious. To prove proposition 4.1, we need to translate this Morse-theoretic intuition over to the formal setup of $\widehat{H F}$.

The first thing to observe is that canceling critical points is not specific to Morse functions: we can do it in any chain complex. More precisely, we have the following lemma.

Lemma 4.4 Suppose $\left(C^{*}, d\right)$ is a chain complex with $C^{i}$ freely generated by $x_{1}, x_{2}, \ldots x_{j_{i}}$ and $C^{i-1}$ freely generated by $y_{1}, y_{2}, \ldots y_{j_{i-1}}$. Denote the $y_{j}$ th component of $d\left(x_{k}\right)$ by $d\left(x_{k}, y_{j}\right)$. Then if $d\left(x_{1}, y_{1}\right)=1,\left(C^{*}, d\right)$ is chain homotopy equivalent to a new complex $\left(\bar{C}^{*}, \bar{d}\right)$. The generators of $\bar{C}^{*}$ are the generators of $C^{*}$, but with $x_{1}$ and $y_{1}$ omitted, and $\bar{d}$ agrees with $d$ except on $\bar{C}^{i}$, where we have

$$
\bar{d}\left(x_{i}\right)=d\left(x_{i}\right)+d\left(x_{i}, y_{1}\right) d\left(x_{1}\right)
$$

This is most likely a folk theorem. A detailed proof may be found in Lemmas $3.7-3.9$ of $[2]$.

To prove the proposition, we want to apply this lemma repeatedly to $\widehat{C F}_{s}(K)$. To do so, we need to show that we still have $d\left(\hat{x}_{2 i}, \hat{x}_{2 i+1}\right)= \pm 1$ in the new complex. This follows from the fact that we have a "Morse function."

Suppose we have chosen a metric $g$ and a basepoint $z_{k}$ on $\Sigma_{2}$. Then for any $i$ and $j$, there is a unique class $\phi_{j}^{i} \in \pi_{2}\left(\hat{x}_{i}, \hat{x}_{j}\right)$ whose domain misses the basepoint. Since

$$
\mathcal{D}\left(\phi_{k}^{i}\right)=\mathcal{D}\left(\phi_{j}^{i}\right)+\mathcal{D}\left(\phi_{k}^{j}\right),
$$

we can choose a function $A:\left\{\hat{x}_{i}\right\} \rightarrow \mathbf{R}$ with $A\left(\hat{x}_{i}\right)-A\left(\hat{x}_{j}\right)$ equal to the signed area of $\mathcal{D}\left(\phi_{j}^{i}\right)$. Holomorphic disks always have positive domains, so $d\left(\hat{x}_{i}, \hat{x}_{j}\right)=0$ unless $A\left(\hat{x}_{i}\right)>A\left(\hat{x}_{j}\right)$. 
Lemma 4.5 For an appropriate choice of metric on $\Sigma_{2}$ the intervals

$$
I_{j}=\left[A\left(\hat{x}_{2 j+1}\right), A\left(\hat{x}_{2 j}\right)\right]
$$

are disjoint.

Proof Since we are working with the stable complex, our basepoint is in the outer part of the spiral. We think of the surface $\Sigma_{2}$ as divided into components by the $\alpha_{i}$ and $\beta_{i}$ and vary the area of each component. Choose the area of the interior region and the spiral to be some very small number $\epsilon$. Thus the length of the interval $I_{j}$ is less than $\epsilon$. If we choose $t_{j} \in I_{j}$, it suffices to show that we can vary the areas of the exterior components so none of the $t_{j}$ are within $2 \epsilon$ of each other.

To do this, we consider the exterior region, which is divided into $(p+1) / 2$ components by $\alpha_{1}$. We label these components $C_{i}$ (starting with the one nearest to the spiral), and take the area of $C_{i}$ to be $M+\eta_{i}-\eta_{i-1}$, where $M \gg \eta_{i} \gg \epsilon$ (except for $\eta_{0}=\eta_{(p+1) / 2}=0$.) Now the domain of $\phi_{2 k-1}^{2 k}$ is the complement of the domain of an exterior differential, so the area of $\mathcal{D}\left(\phi_{2 k-1}^{2 k}\right)$ is a constant plus or minus $\eta_{j_{k}}$ for some $j_{k}$ which is uniquely associated to $k$. Thus if we vary a single $\eta_{j}$ while holding the other $\eta_{i}$ fixed, we vary one of the $t_{k}-t_{k+1}$ by $\pm \eta_{j}$ while holding the other such differences fixed. By varying first the difference $t_{2}-t_{1}$, then the difference $t_{3}-t_{2}$, and so forth, we can make sure all the $t_{i}$ differ by more than $\epsilon$. (This method also shows that we can assume $A\left(x_{1}\right)$ is not in any of the $I_{j}$.)

Proof of Proposition 4.1 We start with the complex $\widehat{C F}_{s}(K)$, which we label $\left(C_{0}^{*}, d_{0}\right)$. By Lemma 4.4 , we can cancel $\hat{x}_{2}$ and $\hat{x}_{3}$ to obtain a new complex $\left(C_{1}^{*}, d_{1}\right)$. We claim that we still have $d_{1}\left(\hat{x}_{2 i}, \hat{x}_{2 i+1}\right)= \pm 1$ for $i>1$. Clearly, it suffices to show that either $d_{0}\left(\hat{x}_{2 i}, \hat{x}_{3}\right)=0$ or $d_{0}\left(\hat{x}_{2}, \hat{x}_{2 i+1}\right)=0$. But since $d_{0}(x, y)=0$ if $A(y)>A(x)$, this follows from the fact that the intervals $I_{1}$ and $I_{i}$ are disjoint.

Moreover, we claim that we still have $d_{1}(x, y)=0$ if $A(y)>A(x)$. To see this, suppose the contrary. Then since $d_{0}(x, y)=0$, we must have $d_{0}\left(x, \hat{x}_{3}\right) \neq 0 \neq$ $d_{0}\left(\hat{x}_{2}, y\right)$, so $A(x)>A\left(\hat{x}_{3}\right)$. In fact $A(x)>A\left(\hat{x}_{2}\right)$, since the intervals $I_{j}$ are all disjoint. Similarly, we have $A\left(\hat{x}_{3}\right)>A(y)$, so

$$
A(x)>A\left(\hat{x}_{2}\right)>A\left(\hat{x}_{3}\right)>A(y)
$$

which contradicts our assumption that $A(y)>A(x)$.

To finish the proof, we simply repeat this process, cancelling $\hat{x}_{4}$ and $\hat{x}_{5}$ to obtain a complex $C_{2}^{*}$, and so on until we reach $C_{(p-1) / 2}^{*}$, which has $x_{1}$ as its 
only generator. Since all the $C_{i}^{*}$ are chain homotopy equivalent, the result follows.

Corollary 4.3 $H F_{s}^{+}(K) \cong \mathbf{Z}\left[u^{-1}\right]$, where the homology class $1 \in \mathbf{Z}\left[u^{-1}\right]$ has the same grading as $\left[x_{1}, 0\right] \in C F_{s}^{+}(K)$.

Proof Follows immediately from the spectral sequence of Lemma 4.2.

Corollary 4.4 $H F_{s}^{-}(K) \cong \mathbf{Z}[u]$, where the homology class 1 has the same grading as $\left[x_{1},-1\right] \in C F_{s}^{-}(K)$.

\subsection{Computing $\mathrm{HF}^{+}(K)$}

To prove Theorem 1, we consider $\widehat{C F}_{s}(K)$ and $C F_{s}^{+}(K)$ as subsets of $C F^{\infty}(K)$. Throughout this section, we describe elements of $C F^{\infty}(K)$ using the bracket notation with respect to a stable $\operatorname{Spin}^{c}$ structure. With this convention, the generators of $\widehat{C F}_{s}(K)$ are the points $\left[x_{i}, 0\right]$, and the generators of $C F_{s}^{+}(K)$ are the points $\left[x_{i}, j\right]$ with $j \geq 0$. We choose an absolute grading on $C F^{\infty}(K)$ which agrees with $\epsilon_{x}$ on $\widehat{C F}_{s}(K)$. From Lemma 3.6, we see that the grading of $\left[x_{1}, 0\right]$ is $\sigma^{\prime}$.

Let us now put $C F^{+}\left(K, \mathbf{s}_{k}\right)$ into the picture as well. Recall that to get the generators of $\widehat{C F}\left(K, \mathbf{s}_{k}\right)$, we reflect the generators of $\widehat{C F}_{s}(K)$ at level $k$. We view the former complex as a subset of $C F^{\infty}(K)$, positioned so that the unreflected generators (those with $\epsilon_{x} \leq k$ ) agree with the corresponding generators of $\widehat{C F}_{s}(K)$. The generators of $C F^{+}\left(K, \mathbf{s}_{k}\right)$ correspond to those generators of $C F^{\infty}(K)$ lying on or above $\widehat{C F}\left(K, \mathbf{s}_{k}\right)$. In particular, they include all the generators of $C F_{s}^{+}(K)$. This situation is illustrated in Figure 10.

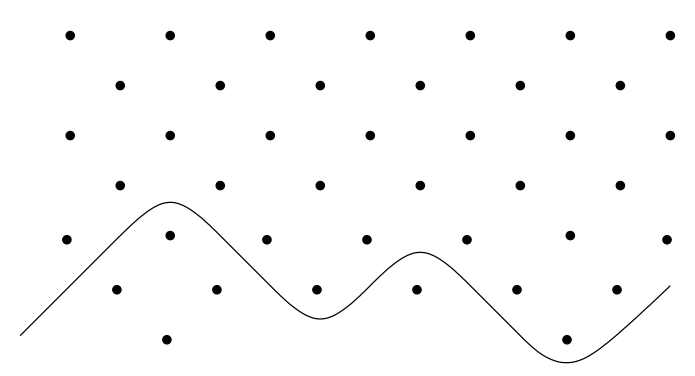

Figure 10: The generators of $C F^{+}\left(K(13,5), \mathbf{s}_{0}\right)$. The subcomplex $C_{0}$ lies below the line, and the quotient complex $C F_{s}^{+}$is above it. 
Let $C_{k}=C F^{+}\left(K, \mathbf{s}_{k}\right) \cap C F_{s}^{-}(K)$. Then $C_{k}$ is a subcomplex of $C F^{+}\left(K, \mathbf{s}_{k}\right)$, since $C F_{s}^{-}(K)$ is a subcomplex of $C F^{\infty}(K)$. Moreover, if we discard the generators of $C_{k}$ from $C F^{+}\left(K, \mathbf{s}_{k}\right)$, we are left with $C F_{s}^{+}(K)$. Thus we have a short exact sequence of chain complexes

$$
0 \longrightarrow C_{k} \longrightarrow C F^{+}\left(K, \mathbf{s}_{k}\right) \longrightarrow C F_{s}^{+}(K) \longrightarrow 0 .
$$

We will compute the homology of $C_{k}$ by comparing it to $C F_{s}^{-}(K)$ and $C F_{a}^{+}(K)$. Since all of the indices in the argument can get a bit confusing, we first describe a specific example. Suppose we wish to compute $\mathrm{HF}^{+}\left(K(13,5), \mathbf{s}_{0}\right)$, so the situation is as shown in Figure 10. $C_{0}$ has generators in three rows, with gradings $0,-1$, and -2 . As shown in Figure 11a, the upper two rows are identical to the top two rows of the complex $C F_{s}^{-}(K)$. Thus to compute the homology in the top row, it suffices to know $H F_{s}^{-}(K)$. Similarly the bottom two rows of $C_{k}$ are the same as the bottom two rows of $C F_{a}^{+}(K)$, and we can compute the homology of the bottom row of $C_{k}$ in terms of $H F_{a}^{+}(K)$.

We now consider the general situation.

Lemma 4.6 The set of generators of $C_{k}$ is $\left\{\left[x_{i}, j\right] \mid 0>j \geq k-\epsilon_{x}\left(x_{i}\right)\right\}$.

Proof We get the complex $\widehat{C F}\left(K, \mathbf{s}_{k}\right)$ by reflecting the complex for $\widehat{C F}_{s}(K)$ at level $k$. Thus the generators of $\widehat{C F}\left(K, \mathbf{s}_{k}\right) \subset C F^{\infty}(K)$ are of the form

$$
\begin{array}{ll}
{\left[x_{i}, k-\epsilon_{x}\left(x_{i}\right)\right]} & \text { if } k \leq \epsilon_{x}\left(x_{i}\right) \\
{\left[x_{i}, 0\right]} & \text { if } k \geq \epsilon_{x}\left(x_{i}\right)
\end{array}
$$

Since $C_{k}$ is generated by those $\left[x_{i}, j\right] \in C F^{+}\left(K, \mathbf{s}_{k}\right)$ with $j<0$, the result follows.

Visually, we can restate this result as follows: to get the complex $C_{k}$, start with the complex $C F_{s}^{-}(K)$, truncate everything below the horizontal line with grading $k-1$, and then add in the reflection about this line, as illustrated in Figure 11. Indeed, truncating at grading $k-1$ gives all the points of the form $\left[x_{i}, j\right]$ with $j<0$ and

$$
\operatorname{gr}\left(\left[x_{i}, j\right]\right)=\epsilon_{x}\left(x_{i}\right)+2 j \geq k-1
$$

or equivalently

$$
0>2 j \geq k-1-\epsilon_{x}\left(x_{i}\right) .
$$

Adding in the reflection gives us all the points $\left[x_{i}, j\right]$ with

$$
0>2 j \geq k-1-\epsilon_{x}\left(x_{i}\right)-\left(-2-\left[k-1-\epsilon_{x}\left(x_{i}\right)\right]\right)=2\left(k-\epsilon_{x}\left(x_{i}\right)\right) .
$$

But this is precisely the condition of the lemma. 
a)

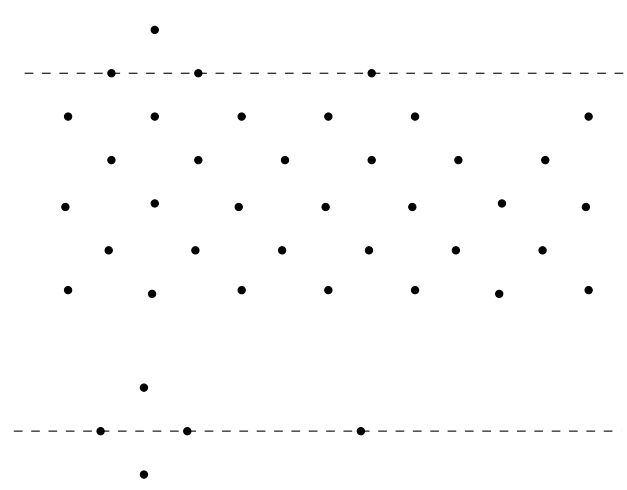

Figure 11: To obtain $C_{0}$ for $K(13,5)$, we (a) truncate $C F_{s}^{-}$at grading -1 and (b) take the union of this set and its reflection.

Corollary 4.5 $H_{i}\left(C_{k}\right) \cong H F_{s i}^{-}(K)$ for $i \geq k$.

Proof For $i$ in this range we are in the top half of $C_{k}$, where the complexes $C_{k}$ and $C F_{s}^{-}(K)$ are identical.

On the other hand, the reflected half of $C_{k}$ is a subcomplex of the antistable complex. Indeed, truncating $\widehat{C F}_{s}(K)$ above level $k+1$ and then reflecting is the same as reflecting $\widehat{C F}_{s}(K)$ to obtain $\widehat{C F}_{a}(K)$ and then truncating below level $-k-1$.

Corollary 4.6 $H_{i}\left(C_{k}\right) \cong H F_{s}^{+}{ }_{i-2 k}(K)$ for $i \leq k-2$.

Proof For $i$ in this range $C_{k}$ and $C F_{a}^{+}(K)$ are the same, so

$$
H_{i}\left(C_{k}\right) \cong H F_{a}^{+}{ }_{i-2 k}(K) \cong H F_{s i-2 k}^{+}(K)
$$

by the charge conjugation symmetry of section 4.2 .

To sum up, we now know $H_{i}\left(C_{k}\right)$ for every $i \neq k-1$. To get $H_{k-1}\left(C_{k}\right)$ we need an easy result from algebra, whose proof is left to the reader:

Lemma 4.7 Suppose

$$
A \stackrel{f}{\longrightarrow} B \stackrel{g}{\longrightarrow} C \oplus D
$$

is a sequence of free abelian groups with $g f=0$ and ker $g / \operatorname{im} f$ free. If $g^{\prime}=$ $\pi g: B \rightarrow C$ is the obvious map, then $\operatorname{ker} g^{\prime} / \operatorname{im} f$ is free as well. 
In particular, if we take $A=C F_{s k}^{-}(K)=\left(C_{k}\right)_{k}, B=C F_{s k-1}^{-}(K)=\left(C_{k}\right)_{k-1}$, and $C \oplus D=\left(C_{k}\right)_{k-2} \oplus D=C F_{s k-2}^{-}(K)$, we see that $H_{k-1}\left(C_{k}\right)$ is a free group. To compute its rank, we use the Euler characteristic.

\section{Lemma 4.8}

$$
\chi\left(C_{k}\right)=(-1)^{\sigma^{\prime}} \sum_{i>k}(i-k) a_{k}=(-1)^{\sigma^{\prime}} u_{k}
$$

Proof Write

$$
C_{k}=\bigcup_{j<0} D_{k, j}
$$

where $D_{k, j}$ contains those elements of $C_{k}$ of the form $\left[x_{i}, j\right]$. From Lemma 4.6, we see that $D_{k, j}$ is isomorphic to the complex $\widehat{C F}_{s}(K)$ truncated at level $k-j$ and translated down by $2 j$. By Proposition 3.5, it contributes

$$
(-1)^{\sigma^{\prime}} \sum_{i \geq k-j} a_{j}
$$

to the Euler characteristic. Summing up these contributions for all $j<0$ gives the desired result.

Putting these facts together, we obtain the following:

Proposition 4.2 For $k \geq 0$, there is an isomorphism of $\mathbf{Z}[u]$ modules

$$
H_{*}\left(C_{k}\right) \cong Q_{k} \oplus V_{k}
$$

where $Q_{k}$ and $V_{k}$ are as in the statement of Theorem 1, except that if $\sigma \geq 0$ the grading of $u^{-h_{k}+1} \in V_{k}$ is $\sigma^{\prime}-2$.

Proof Suppose $\sigma \geq 0$. Then for $i<k-1$ we have $H_{i}\left(C_{k}\right) \cong H F_{s}^{+}{ }_{i-2 k}(K)=$ 0 . Indeed, the latter group vanishes whenever $i-2 k<\sigma^{\prime}$, and the assumption $k \geq 0$ implies $i-2 k<0$. Similarly, Corollary 4.5 implies that for $i>k-1$, $H_{i}\left(C_{k}\right)=\mathbf{Z}$ for $i=\sigma^{\prime}-2, \sigma^{\prime}-4$, and so on down to $i=k$ or $i=k+1$ (depending on the parity of $\sigma^{\prime}$ ) and that the other homology groups are trivial.

To get $H_{k-1}\left(C_{k}\right)$, we recall that it is free (by Lemma 4.7$)$ and use Lemma 4.8 to compute its rank. We find that $H_{k-1}\left(C_{k}\right)=\mathbf{Z}^{\left|b_{k}\right|}$ if $k \equiv \sigma^{\prime} \bmod 2$ and $H_{k-1}\left(C_{k}\right)=\mathbf{Z}^{\left|b_{k}\right|+1}$ otherwise. This proves the statement at the level of groups.

To check it at the level of modules, we note that the $u$ action in the top half of $C_{k}$ clearly agrees with the $u$ action on $H F_{s}^{-}(K)$. In other words, it sends 
the generator in dimension $\sigma^{\prime}-2 i$ to the generator in dimension $\sigma^{\prime}-2(i+1)$. In view of the structure of $H_{*}\left(C_{k}\right)$, it now suffices to show that if $k<\sigma^{\prime}$ and $k-1 \equiv \sigma^{\prime} \bmod 2$, then $u$ takes the generator of $H_{k+1}\left(C_{k}\right)$ to a nonzero element of $H_{k-1}\left(C_{k}\right)$. But this follows from the fact that the $u$ action commutes with the inclusion $H F_{s}^{-}{ }_{i}(K) \hookrightarrow H_{i}\left(C_{k}\right)$ for $i \geq k-1$.

This proves the proposition in the case $\sigma \geq 0$. When $\sigma \leq 0$ the roles of the bottom and top halves are reversed, but the proof is otherwise the same.

To prove Theorem 1, we use the long exact sequence:

$$
\cdots H_{i}\left(C_{k}\right) \longrightarrow H^{+}{ }_{i}\left(K, \mathbf{s}_{k}\right) \stackrel{\pi_{*}}{\longrightarrow} H F_{s}^{+}{ }_{i}(K) \longrightarrow H_{i-1}\left(C_{k}\right) \quad \cdots
$$

We claim that the map $\pi_{*}$ is always surjective. This is clearly true when $i$ is very large. But this implies the claim for all $i$, since $H F_{s}^{+}(K) \cong \mathbf{Z}\left[u^{-1}\right]$ and the maps in the long exact sequence respect the $u$ action.

It is now a simple matter to check that the theorem holds at the level of groups. To verify the module structure, we consider the cases $\sigma(K) \geq 0$ and $\sigma(K) \leq 0$ separately.

Case $1 \sigma(K) \geq 0$ : From the short exact sequence of $\mathbf{Z}[u]$ modules

$$
0 \longrightarrow H_{*}\left(C_{k}\right) \longrightarrow H F_{*}^{+}\left(K, \mathbf{s}_{k}\right) \longrightarrow H F_{s}^{+}(K) \longrightarrow 0
$$

we see that $H F_{*}^{+}\left(K, \mathbf{s}_{k}\right)$ must have a summand $\mathbf{Z}\left[u^{-1}\right]$ which maps onto $H F_{s *}^{+}(K)$. We claim that the kernel of this map is isomorphic to $V_{k}$. Indeed, the kernel can hardly be larger than $V_{k}$, since there is no such module available in $H_{*}\left(C_{k}\right)$. To get other direction, we observe that $C F_{i}^{+}\left(K, \mathbf{s}_{k}\right)$ is identical to $C F_{i}^{\infty}(K)$ for $i \geq k-1$. Indeed, the largest $j$ for which $\left[x_{i}, j\right] \notin C F^{+}\left(K, \mathbf{s}_{k}\right)$ is -1 if $k \geq \epsilon_{x}\left(x_{i}\right)$ and $k-\epsilon_{x}\left(x_{i}\right)-1$ if $k \leq \epsilon_{x}\left(x_{i}\right)$. In both cases, $\operatorname{gr}\left[x_{i}, j\right]<k-1$. It follows $H F_{*}^{+}\left(K, \mathbf{s}_{k}\right)$ has a $\mathbf{Z}\left[u^{-1}\right]$ summand which extends at least as far down in the grading as $V_{k}$. Together with the known structure of $C F_{s}^{+}(K)$, this implies that the kernel is at least as big as $V_{k}$, thus proving the claim. It is now easy to see that to see that the $\mathbf{Z}[u]$ module structure must be as described.

Case $2 \sigma(K) \leq 0$ : As in the previous case, $H F_{*}^{+}\left(K, \mathbf{s}_{k}\right)$ must have a summand $\mathbf{Z}\left[u^{-1}\right]$ which maps onto $H F_{s *}^{+}(K)$. This time, however, it is easy to show that this map has no kernel. Indeed, since $\sigma \leq 0$ and $k \geq 0$, there are no elements in $H_{*}\left(C_{k}\right)$ with grading less than that of $1 \in H F_{s *}^{+}(K) \cong \mathbf{Z}\left[u^{-1}\right]$. Thus the short exact sequence splits as a sequence of $\mathbf{Z}[u]$ modules. 
This finishes the proof of Theorem 1. Corollary 4.1 is an immediate consequence of the theorem and Lemma 4.1, while Corollary 4.2 follows from the discussion of $H F^{\text {red }}$ in section 4 of [6].

\section{Using the exact triangle}

In this section, we describe how to calculate $H F^{+}\left(K^{n}\right)$ for any $n$. The results may be summarized as follows:

Proposition 5.1 For $k \neq 0$, there is an isomorphism of $\mathbf{Z}[u]$ modules

$$
H F^{+}\left(K^{0}, \mathbf{s}_{k}\right) \cong Q_{k} \oplus V_{k} .
$$

Similarly, if we use the twisted coefficients of [7] we have

$$
\underline{H F^{+}}\left(K^{0}, \mathbf{s}_{0}\right) \cong\left(Q_{0} \oplus V_{0}\right) \otimes \mathbf{Z}\left[T, T^{-1}\right] \oplus \mathbf{Z}\left[u^{-1}\right] .
$$

Proposition 5.2 For $n \in \mathbf{Z}^{+}$and $\sigma(K) \leq 0$, we have

$$
H F^{+}\left(K^{n}, \mathbf{s}_{k}\right) \cong \mathbf{Z}\left[u^{-1}\right] \oplus \bigoplus_{i \equiv k(n)} Q_{i} \bigoplus_{i \equiv k(n)} V_{i}
$$

while if $\sigma(K) \geq 0$

$$
H F^{+}\left(K^{n}, \mathbf{s}_{k}\right) \cong \mathbf{Z}\left[u^{-1}\right] \oplus \bigoplus_{i \equiv k(n)} Q_{i} \bigoplus_{i_{0} \neq i \equiv k(n)} V_{i}
$$

where $i_{0}$ is the representative of $k \bmod n$ with the smallest absolute value.

\section{Remarks}

(1) The groups $Q_{k}$ and $V_{k}$ are described in the statement of Theorem 1. By convention, $Q_{-k}=Q_{k}$ and $V_{-k}=V_{k}$.

(2) For the moment, we consider all these groups to be $\mathbf{Z} / 2$ graded. The absolute grading of [8] can easily be worked out from the exact triangle as well.

(3) We can use the same methods as in the large $n$ case to compute the groups $\widehat{H F}\left(K^{n}, \mathbf{s}_{k}\right)$ and $H F^{-}\left(K^{n}, \mathbf{s}_{k}\right)$. To get $H F^{+}\left(K^{-n}, \mathbf{s}_{k}\right)$ we use the isomorphism

$$
H F^{+}\left(K^{-n}, \mathbf{s}_{k}\right) \cong\left(H F^{-}\left(\bar{K}^{n}, \mathbf{s}_{k}\right)\right)^{*} .
$$


(4) These results are summarized in the following rule of thumb: the Floer homology of the knot $K$ behaves like the sum of a fixed group $Q$ and the Floer homology of the $(2, n)$ torus knot of the same signature. $Q$ does not interact with $\mathrm{HF}^{+}\left(S^{3}\right)$ in the exact triangle.

To prove these two propositions, we use the exact triangle of [7]. Starting with our knowledge of $H^{+}\left(K^{n}, \mathbf{s}_{k}\right)$ for $n \gg 0$, we use the exact triangle to calculate $H F^{+}\left(K^{0}, \mathbf{s}_{k}\right)$. Then we apply the triangle again to get $H F^{+}\left(K^{n}\right)$ for any $n$.

\subsection{Review of the exact triangle}

The exact triangle - first conceived by Floer and now widened in scope by Ozsváth and Szabó - has numerous incarnations. The one we want is described in Theorem 10.19 of [7].

Theorem 5.1 For $n \in \mathbf{Z}^{+}$, there are exact triangles

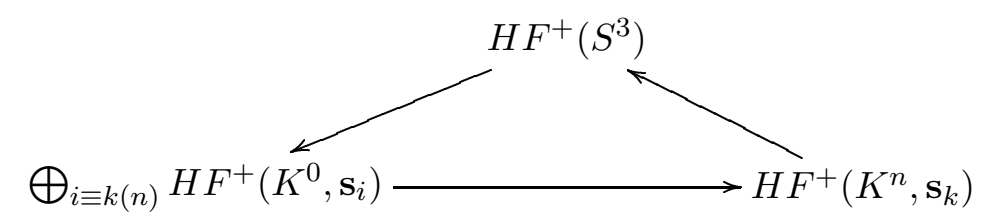

and

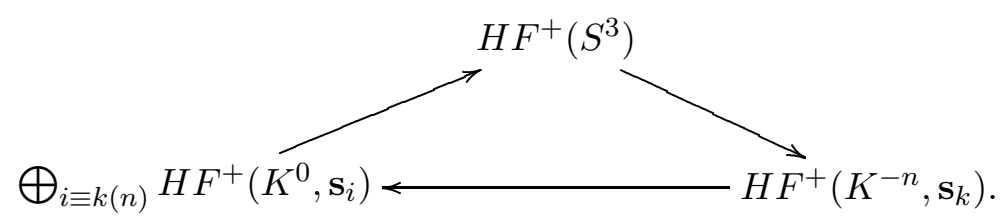

All the maps in these triangles respect the $\mathbf{Z}[u]$ module structure.

\section{Remarks}

(1) Note that for $n \geq 2 g-1$, the adjunction inequality implies that at most one term in the direct sum is nontrivial.

(2) Our notation for $\operatorname{Spin}^{c}$ structures is as follows. In the language of section 3.1, $H^{2}\left(K^{0}\right)$ is generated by $A_{1}^{*}$ (the Poincare dual of $A_{1}$ ). On $K^{0}$, we let $\mathbf{s}_{k}$ be the $\operatorname{Spin}^{c}$ structure whose determinant line bundle has first Chern class $2 k A_{1}^{*}$. We have to check that for $n \gg 0$, the $\operatorname{Spin}^{c}$ structure $\mathbf{s}_{k}$ on $K^{0}$ really does induce $\mathbf{s}_{k}$ on $K^{n}$. To see this, note that on $K^{n}$, the difference $\mathbf{s}_{k+1}-\mathbf{s}_{k}=2 A_{1}^{*}$. It follows that there is a constant $m$ such 
that the $\operatorname{Spin}^{c}$ structure $\mathbf{s}_{k}$ on $K^{0}$ induces $\mathbf{s}_{k+m}$ on $K^{n}$. By adjunction, $H F^{+}\left(K^{0}, \mathbf{s}_{k}\right)$ is nontrivial only when $-g<k<g$. On the other hand, we know that $H F^{+}\left(K, \mathbf{s}_{k}\right) \neq H F^{+}\left(S^{3}\right)$ precisely for $-g<k<g$, so we must have $m=0$. (This proves that $\mathbf{s}_{k}$ and $\mathbf{s}_{-k}$ are conjugate $\operatorname{Spin}^{c}$ structures on $K^{n}$, as mentioned in section 4.2.)

(3) To use the second exact triangle, we need the following lemma.

Lemma 5.1 For $n \gg 0$,

$$
H F^{+}\left(K^{-n}, \mathbf{s}_{k}\right) \cong \begin{cases}\mathbf{Z}\left[u^{-1}\right] \oplus Q_{k} \oplus V_{k} & \text { if } \sigma \geq 0 \\ \mathbf{Z}\left[u^{-1}\right] \oplus Q_{k} & \text { if } \sigma \leq 0\end{cases}
$$

Proof Observe that

$$
H F^{+}\left(K^{-n}, \mathbf{s}_{k}\right) \cong H F_{-}\left(-K^{-n}, \mathbf{s}_{k}\right) \cong\left(H F^{-}\left(\bar{K}^{n}, \mathbf{s}_{k}\right)\right)^{*}
$$

where $\bar{K}$ is the mirror image of $K$. (In the last equality, we have used the fact that $H F^{-}\left(\bar{K}^{n}, \mathbf{s}_{k}\right)$ is free over $\mathbf{Z}$.) Since $K$ and $\bar{K}$ have the same Alexander polynomials and $\sigma(\bar{K})=-\sigma(K)$, the lemma follows from Corollary 4.2.

\subsection{Proof of Proposition 5.1}

We use the exact triangle for $n \gg 0$. Consider the map $h: H F^{+}\left(K^{n}, \mathbf{s}_{k}\right) \rightarrow$ $H F^{+}\left(S^{3}\right) \cong \mathbf{Z}\left[u^{-1}\right]$. For $k \neq 0, H F^{+}\left(K^{0}, \mathbf{s}_{k}\right)$ is finitely generated, so $h$ must be onto when the grading is large. The only way this can happen is if the $\mathbf{Z}\left[u^{-1}\right]$ summand in $H F^{+}\left(K^{n}, \mathbf{s}_{k}\right)$ maps onto $H F^{+}\left(S^{3}\right)$. In this case, ker $h$ is a torsion module $R=\mathbf{Z}\left[u^{-1}\right] / u^{-m}$ plus the other summands in $H F^{+}\left(K^{n}, \mathbf{s}_{k}\right)$.

Suppose that $\sigma(K) \geq 0$, so that $H F^{+}\left(K^{n}, \mathbf{s}_{k}\right) \cong \mathbf{Z}\left[u^{-1}\right] \oplus Q_{k}$. Then

$$
H F^{+}\left(K^{0}, \mathbf{s}_{k}\right) \cong Q_{k} \oplus R \text {. }
$$

But by Theorem 9.1 of [7] we have $\chi\left(H F^{+}\left(K^{0}, \mathbf{s}_{k}\right)\right)=u_{k}=\chi\left(H_{*}\left(C_{k}\right)\right)$. It follows that we must have $R \cong V_{k}$. Similarly, if $\sigma(K) \leq 0$, we see that $H F^{+}\left(K^{0}, \mathbf{s}_{k}\right) \cong Q_{k} \oplus V_{k} \oplus R$. In this case, the Euler characteristic shows that the group $R$ must be trivial.

The case $k=0$ requires some special attention, since $H F^{+}\left(K^{0}, \mathbf{s}_{0}\right)$ is not finitely generated. To get around this problem, we use the exact triangle for twisted coefficients (Theorem 10.21 of [7]). In this case, we have a map

$$
h: H F^{+}\left(K^{n}, \mathbf{s}_{0}\right)\left[T, T^{-1}\right] \rightarrow H F^{+}\left(S^{3}\right)\left[T, T^{-1}\right] \cong \mathbf{Z}\left[u^{-1}\right]\left[T, T^{-1}\right] .
$$


We consider the action of $h$ on the $\mathbf{Z}\left[u^{-1}\right]$ summand of $H F^{+}\left(K, \mathbf{s}_{0}\right)$. Clearly $h\left(u^{-k}\right)$ is nonzero - otherwise $\underline{H F^{+}}\left(K^{0}, \mathbf{s}_{0}\right)$ would contain a copy of $\mathbf{Z}\left[u^{-1}\right]\left[T, T^{-1}\right]$, which it does not. Thus we can write

$$
h\left(u^{-k}\right)=F(T) u^{-k+m}+(\text { lower order terms })
$$

for some $m \geq 0$. We claim that after a change of basis in $H F^{+}\left(S^{3}\right)\left[T, T^{-1}\right.$, we have $F(T)=T-1$. This follows from the calculation of $H F^{\infty}$ in [7]. Indeed, the fact that $\underline{H F^{\infty}}\left(K^{0}\right) \cong \mathbf{Z}\left[u, u^{-1}\right]$ implies that (after a change of basis) $F$ is a polynomial of degree 1 . On the other hand, to get $h$ in the untwisted triangle, we substitute $T=1$ in the twisted map. Since $H F^{\infty}\left(K^{0}\right) \cong H^{*}\left(T^{2}\right) \otimes \mathbf{Z}\left[u, u^{-1}\right]$, we must have $F(1)=0$.

Now that we understand the action of $h$, we see that

$$
\underline{H F^{+}}\left(K^{0}, \mathbf{s}_{0}\right) \cong\left(Q_{0} \oplus R\right) \otimes \mathbf{Z}\left[T, T^{-1}\right] \oplus \mathbf{Z}\left[u^{-1}\right]
$$

where $R$ is the torsion module $\mathbf{Z}\left[u^{-1}\right] / u^{-m}$. Although it is not explicitly stated in [7], it is not difficult to follow through the proof of Theorem 9.1 there and see that it applies to $\underline{H F^{+}}\left(K^{0}, \mathbf{s}_{0}\right)$ as well, so long as we take the Euler characteristic of $\underline{H F^{+}}\left(K^{0}, \mathbf{s}_{0}\right)$ as a $\mathbf{Z}\left[T, T^{-1}\right]$ module. We can now argue as in the case $k \neq 0$.

\subsection{Proof of Proposition 5.2}

To compute $H F^{+}\left(K^{n}, \mathbf{s}_{k}\right)$, we use the exact triangle

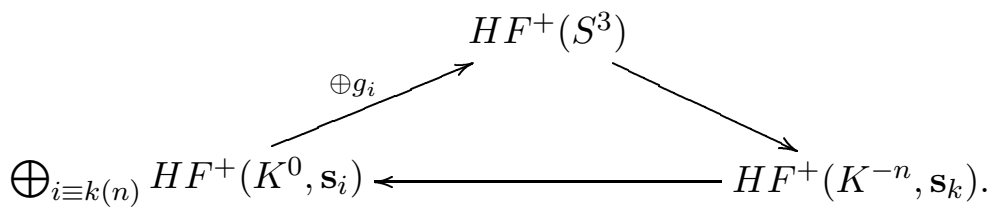

The key point is that the maps $g_{i}: H F^{+}\left(K^{0}, \mathbf{s}_{i}\right) \rightarrow H F^{+}\left(S^{3}\right)$ are independent of $n$. Indeed $g_{i}$ is simply the map induced by the standard cobordism between $S^{3}$ and $K^{0}$.

Lemma 5.2 For $i \neq 0$, the map $g_{i}$ is trivial if $\sigma \geq 0$. If $\sigma \leq 0, g_{i}$ maps the summand $V_{i}$ onto the span of $\left\{1, u^{-1}, u^{-2}, \ldots, u^{-h_{i}+1}\right\}$ in $H F^{+}\left(S^{3}\right)$. If we use twisted coefficients, these statements are true for $i=0$ as well. With untwisted coefficients, $g_{0}$ maps $H F^{+}\left(K^{0}, \mathbf{s}_{0}\right)$ onto $H F^{+}\left(S^{3}\right)$ with kernel $Q_{0} \oplus V_{0} \oplus \mathbf{Z}\left[u^{-1}\right]$. 
Proof Suppose $i \neq 0$ and take $n \gg 0$. The usual argument shows that $H F^{+}\left(S^{3}\right)$ must map onto the $\mathbf{Z}\left[u^{-1}\right]$ summand in $H F^{+}\left(K^{-n}, \mathbf{s}_{k}\right)$, with a $\mathbf{Z}[u]$ torsion module $R$ as kernel. For $\sigma \geq 0$, we have $H F^{+}\left(K^{-n}, \mathbf{s}_{k}\right) \cong Q_{k} \oplus V_{k} \oplus$ $\mathbf{Z}\left[u^{-1}\right]$ by Lemma 5.1. On the other hand, we know $H F^{+}\left(K^{0}, \mathbf{s}_{i}\right) \cong Q_{k} \oplus V_{k}$, so $R$ must be trivial. Likewise, if $\sigma \leq 0$, we know $H F^{+}\left(K^{-n}, \mathbf{s}_{k}\right) \cong Q_{k} \oplus \mathbf{Z}\left[u^{-1}\right]$, so we must have $R \cong V_{k}$. For $i=0$, the same arguments apply if we use twisted coefficients.

The statement for untwisted coefficients is just a rephrasing of the fact that the map $H_{F}^{+}\left(S^{3}\right) \rightarrow H F^{+}\left(K^{-n}, \mathbf{s}_{0}\right)$ is trivial.

It is now elementary to compute $H F^{+}\left(K^{-n}, \mathbf{s}_{k}\right)$. For example, if $\sigma \geq 0$ and $k \not \equiv 0 \bmod n$, all the $g_{i}$ vanish, and we have

$$
H F^{+}\left(K^{-n}, \mathbf{s}_{k}\right) \cong \mathbf{Z}\left[u^{-1}\right] \oplus \bigoplus_{i \equiv k(n)} Q_{i} \bigoplus_{i \equiv k(n)} V_{i} .
$$

On the other hand, when $\sigma \leq 0$, the image of $\oplus g_{i}$ is isomorphic to $V_{k_{0}}$, where $k_{0}$ is the representative of $k \bmod n$ with the smallest absolute value. Thus

$$
H F^{+}\left(K^{-n}, \mathbf{s}_{k}\right) \cong \mathbf{Z}\left[u^{-1}\right] \oplus \bigoplus_{i \equiv k(n)} Q_{i} \bigoplus_{k_{0} \neq i \equiv k(n)} V_{i} .
$$

We leave it to the reader to check that these results also hold in the case $k \equiv 0$ $\bmod n$.

Finally, to get Proposition 5.2, we use the isomorphism

$$
H F^{+}\left(K^{-n}, \mathbf{s}_{k}\right) \cong\left(H F^{-}\left(\bar{K}^{n}, \mathbf{s}_{k}\right)\right)^{*}
$$

together with the usual exact sequence relating $H F^{+}$and $H F^{-}$.

\subsection{Frøyshov's Invariant}

We conclude by justifying the statement about Frøyshov's invariant given in the introduction. In [8], Ozsváth and Szabó define an invariant $d$ which is the analogue (up to a factor of 2 ) of the $h$-invariant in their theory.

To compute $d\left(K^{n}\right)$, we use the fact that the degree of the map $h: H F^{+}\left(S^{3}\right) \rightarrow$ $H F^{+}\left(K^{-n}, \mathbf{s}_{k}\right)$ is determined by purely homological information, namely the values of $n$ and $k$. In particular, for $n=1$, the degree of $h$ is 0 .

Consider the exact triangle for $K^{-1}$. If we use twisted coefficients, the facts stated above imply that $d\left(K^{-1}\right)=2 \operatorname{dim} \operatorname{ker} h$. From Lemma 5.2, we know that

$$
\operatorname{dim} \operatorname{ker} h= \begin{cases}0 & \text { if } \sigma \geq 0 \\ \left|V_{0}\right|=\left\lceil\left|\sigma^{\prime}\right| / 2\right\rceil & \text { if } \sigma \leq 0 .\end{cases}
$$


This gives us $d\left(K^{-1}\right)$. To get $d\left(K^{1}\right)$, we use the easily proved fact that $d(-Y)=$ $-d(Y)$ and the identification $K^{1}=-\bar{K}^{-1}$.

\section{Other Knots}

We conclude by discussing the extent to which the methods used in this paper extend to knots with more than two bridges. Of course there is no analogue of Schubert's theorem for these knots, and thus no explicit general form for their Heegard splitting. On the other hand, given a particular knot, it is straightforward to find a Heegard splitting of its complement. As in the case of two-bridge knots, it is convenient to consider $n$-surgery on $K$ for $n \gg 0$. In this case, we still have a stable complex $\widehat{C F}_{s}(K)$, with an Alexander grading which can be computed by Fox calculus. As in section 3.3, it is straightforward to determine $\widehat{C F}\left(K, \mathbf{s}_{k}\right)$ from $\widehat{C F}_{s}(K)$. In addition, we should still be able to run the argument of section 4.3 to compute the grading of the generator of $\widehat{C F}_{s}$.

The calculation of section 4.4 fails, however, because for a general knot the Alexander grading on $\widehat{C F}_{s}(K)$ does not agree with the Maslov grading. (For example, it is easy to see that the two differ for non two-bridge torus knots.) We refer to those knots for which the two gradings coincide as perfect knots. We expect that the methods of this paper will extend to perfect knots in general, although it is more difficult to compute $\mathrm{HF}_{a}^{+}(K)$. We hope to discuss the issues described above, as well as some other classes of perfect knots, in a future paper.

\section{References}

[1] G. Burde and H. Zieschang, Knots, de Grüyter Studies in Mathematics, No. 5, Walter de Grüyter and Co., Berlin, 1985.

[2] A. Floer, Witten's complex and infinite dimensional Morse theory, J. Differential Geom. 30 (1989), 207-21.

[3] K. Frøyshov, Lectures on Floer homology at Harvard, Spring 2001.

[4] C. McMullen, The Alexander polynomial of a 3-manifold and the Thurston norm on cohomology, Ann. Scient. Ec. Norm. Sup., to appear.

[5] K. Murasugi, Knot theory and its applications, Birkhauser, Boston, 1996.

[6] P.S. Ozsváth and Z. Szabó, Holomorphic disks and topological invariants for homology three-spheres, arXiv:math.SG/0101206

[7] P.S. Ozsváth and Z. Szabó, Holomorphic disks and three-manifold invariants: properties and applications, arXiv:math.SG/0105202 
[8] P.S. Ozsváth and Z. Szabó, Absolutely graded Floer homologies and intersection forms for four-manifolds with boundary, arXiv:math.SG/0110170

[9] H. Schubert, Knoten mit zwei Brücken, Math. Z. 65 (1956), 133-70.

Department of Mathematics, Harvard University

Cambridge, MA 02138, USA

Email: jacobr@math.harvard.edu

Received: 9 April 2002

Algebraic 6 Geometric Topology, Volume 2 (2002) 